\title{
The hp finite element method for problems in mechanics with boundary layers
}

\section{Report}

\section{Author(s):}

Schwab, Christoph; Suri, M.; Xenophontos, C.

Publication date:

1996-10

Permanent link:

https://doi.org/10.3929/ethz-a-004284636

Rights / license:

In Copyright - Non-Commercial Use Permitted

Originally published in:

SAM Research Report 1996-20 


\title{
The $h p$ finite element method for problems in mechanics with boundary layers *
}

\author{
C. Schwab, M. Suri ${ }^{1}$ and C. Xenophontos ${ }^{2}$
}

Research Report No. 96-20

October 1996

Seminar für Angewandte Mathematik

Eidgenössische Technische Hochschule

CH-8092 Zürich

Switzerland

\footnotetext{
* The work of the first two authors was supported in part by the Air Force Office of Scientific Research, Air Force Systems Command, USA Funder Grant F49620-95-I-0230.

${ }^{1}$ Department of Mathematics Statistics, University of Maryland Baltimore County, 101 Hilltop Circle, Baltimore MD 21250, USA

${ }^{2}$ Ohio State University, 4240 Campus Drive, Lima, OH 45804, USA
} 


\title{
The $h p$ finite element method for problems in mechanics with boundary layers *
}

\author{
C. Schwab, M. Suri ${ }^{1}$ and C. Xenophontos ${ }^{2}$ \\ Seminar für Angewandte Mathematik \\ Eidgenössische Technische Hochschule \\ CH-8092 Zürich \\ Switzerland
}

Research Report No. 96-20 October 1996

\begin{abstract}
We consider the numerical approximation of boundary layer phenomena occuring in many singularly perturbed problems in mechanics, such as plate and shell problems. We present guidelines for the effective resolution of such layers in the context of exisiting, commercial $p$ and $h p$ finite element (FE) version codes. We show that if high order, "spectral" elements are available, then just two elements are sufficient to approximate these layers at a near-exponential rate, independently of the problem parameters thickness or Reynolds number. We present $h p$ mesh design principles for situations where both corner singularities and boundary layers are present.
\end{abstract}

\footnotetext{
*The work of the first two authors was supported in part by the Air Force Office of Scientific Research, Air Force Systems Command, USA Funder Grant F49620-95-I-0230.

${ }^{1}$ Department of Mathematics Statistics, University of Maryland Baltimore County, 101 Hilltop Circle, Baltimore MD 21250, USA

${ }^{2}$ Ohio State University, 4240 Campus Drive, Lima, OH 45804, USA
} 


\section{Introduction}

Boundary layer phenomena are well-known in the study of fluid flow problems. Perhaps less known, but equally ubiquitous, is their existence in thermal, plate and shell analysis, where they also play a very significant role. In this paper, we will discuss the properties of boundary layers occurring in problems of plate and shell modeling, and in heat transfer, and address the question of their effective numerical resolution by the $h p$ finite element method.

To motivate our discussion, we begin with a computational experiment from [8]. Consider a thin rectangular plate of size $3 L \times L$, and thickness $d=L / 100$ (See Figure 1 ). $\mathrm{AB}, \mathrm{BC}$ lie on lines of symmetry, AD lies on a line of antisymmetry, and CD is free. The material coefficients shown are those of an aluminum alloy and the shear correction factor is taken as $\widetilde{\kappa}=5 / 6$. A sinusoidal distributed load $q=-\sin (\pi y / 2 L)$ is applied to the plate.

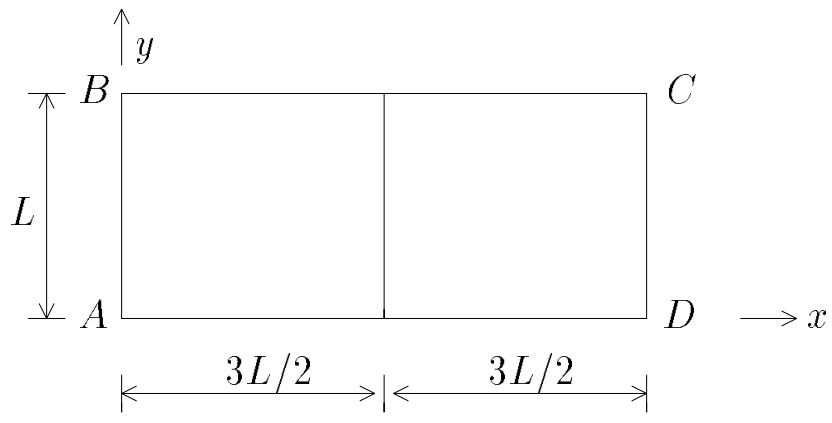

a) Mesh $1 \quad \begin{aligned} E & =10.1 \times 10^{6} \\ \nu & =0.3 .65\end{aligned}$

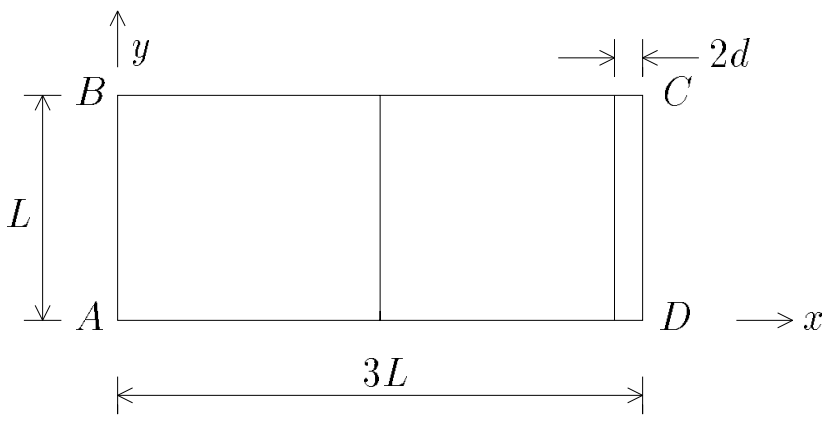

b) Mesh 2

Figure 1: Finite element meshes on rectangular plate 
We analyze the plate using the $p$ version ${ }^{1}$. The analysis is carried out on a ReissnerMindlin (RM) formulation using two different meshes shown in Figure 1. Using product spaces, the polynomial degree $p$ is increased from $p=1$ to $p=8$. For the latter, 400 degrees of freedom are employed in each mesh. Figure 2 shows the computed shear force distribution $Q_{y}(x, 0)$ along the antisymmetric edge from $x=2.968 \mathrm{~L}$ to $3.0 \mathrm{~L}$, obtained with each of the two meshes for $p=8$. It is seen that there is a large discrepancy in the results obtained : the value of $Q_{y}(3,0)$ predicted by Mesh 1 is over 5 times smaller than that predicted by Mesh 2 . The true solution $Q_{y}(x, 0)$ behaves essentially like Figure $2 \mathrm{~b}$ ), i.e. it exhibits a boundary layer effect. The reason for the discrepancy is that uniform grids like Mesh 1 are not very effective in capturing such effects; what is needed are specially designed meshes like Mesh 2.

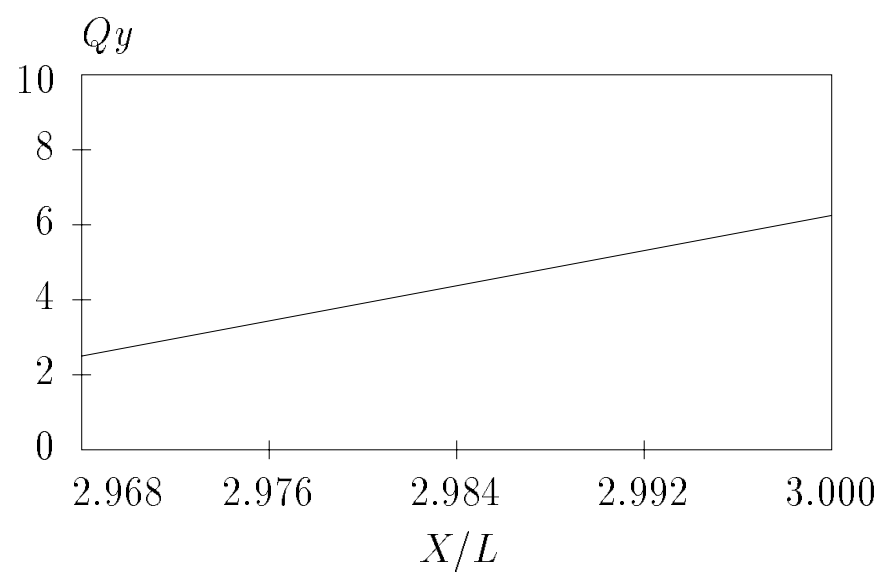

a) Mesh 1

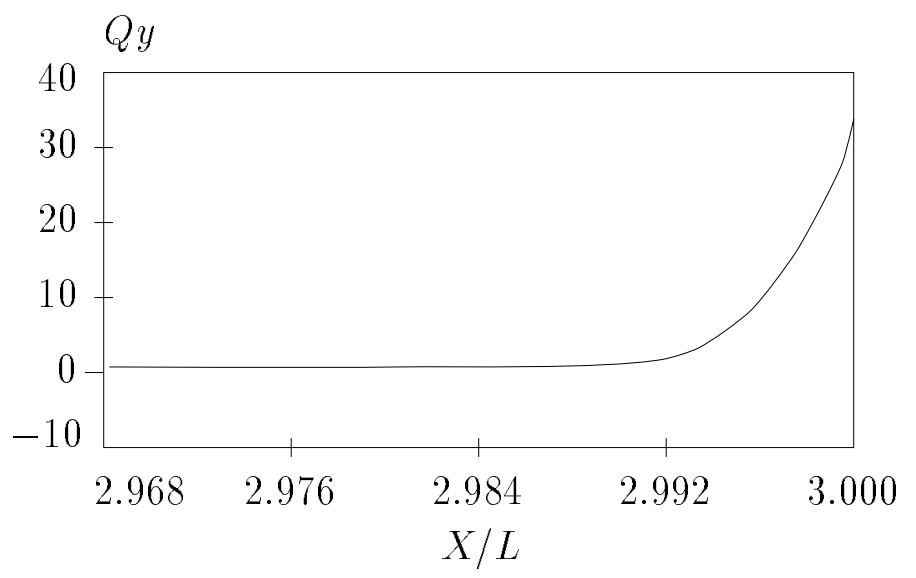

b) Mesh 2

Figure 2: Shear force distribution along line of antisymmetry

\footnotetext{
${ }^{1}$ All computations in this paper (except the one-dimensional ones in Section 2) were performed using STRESS CHECK (ESRD, St. Louis, MO).
} 
The above experiment shows that the solution of plate (and shell) models in solid mechanics and viscous flow in fluid mechanics generally contain boundary layers. These are rapidly varying components which decay exponentially with respect to distance from the edge. More precisely, these are functions of the form

$$
\xi(x, y)=\tau(x, y) e^{-\alpha \rho / d}
$$

where $\tau(x, y)$ is some smooth function, $\rho$ is the distance from the boundary of the plate, $d$ is e.g. the plate thickness, and $\alpha$ is a positive constant (e.g. for the RM plate model, $\alpha=(12 \tilde{\kappa})^{1 / 2}$ where $\tilde{\kappa}$ is the shear correction factor). The exponential term in (1) causes engineering data computed at the boundary to have a high rate of change. As a result, many important design-relevant quantities such as reactions, moments and shear forces may assume their maximum on the edge or within a distance of a few times the thickness of the edge. Finite element solutions must estimate these maxima accurately and reliably, and must therefore be able to "capture" these boundary layers.

With the traditional $h$ version, the usual procedure to resolve the layers is to refine the mesh sufficiently in a strip inside the boundary, so that the mesh width in this region satisfies a relation like $h<C d$. A more efficient procedure is to perform the refinement in a non-uniform way, as is done e.g. in [5], [9], [20]. The highest convergence rate possible with these approaches is $O\left(h^{p}\right)$ (in the energy norm), where $p$ is the polynomial degree.

If $p$ (or $h p$ ) capability is also available, then faster (up to exponential) rates of convergence can be realized. Our goal in this paper is to describe the proper design of meshes and selection of polynomial degrees to attain these high convergence rates in the presence of boundary layers.

In Section 2, we explain the basic idea, from [18], in the simplified one-dimensional context. In Section 3, these ideas are used to approximate two-dimensional problems over smooth domains where the solution is smooth except for boundary layers. We consider a model heat transfer problem and a RM plate problem, describing the boundary layers present and showing how near-exponential rates can be attained in practice.

In Section 4, we consider the case when the presence of corners in the domain gives rise to corner singularities in the solution. The exact coupling between these singularities and the boundary layer functions is described for the heat transfer problem over a square. Using results from [21], we show how the FE-mesh should be designed to treat both types of components and obtain near-exponential convergence using the $h p$ FEM. We also describe the meshes that should be used for the heat transfer and RM problems when the domain is a general polygon. Extensions to shell modeling are briefly addressed in Section 5.

Let us describe some notation. For an open, bounded, simply connected set $\Omega \subset \mathbb{R}^{n}$, $n=1,2, H^{\ell}(\Omega)$ will denote the (Sobolev) space of functions with $\ell$ generalized derivatives, with norm $\|\cdot\|_{\ell, \Omega}$ and seminorm $|\cdot|_{\ell, \Omega}$. Let $\left\{\mathcal{T}_{N}\right\}$ be a sequence of partitions of $\Omega$ into elements, parametrized by $N$. The elements $K_{i}, i=1,2, \ldots, M_{N}$ are intervals for $n=1$ and assumed to be a combination of (curvilinear) triangles and quadrilaterals for $n=2$. Let $\hat{I}=(-1,1), \hat{T}=\{(x, y): 0<x<1,0<y<1-x\}, \hat{Q}=(-1,1)^{2}$ represent the standard interval, triangle and quadrilateral respectively. Then we assume that each $K_{i}$ is the image of one of these under a smooth mapping $\mathcal{F}_{i}$. 
For $p=1,2, \ldots$ and $\widehat{K}=\widehat{I}, \widehat{T}$ or $\widehat{Q}$ let $\mathcal{P}_{p}(\widehat{K})$ denote the set of polynomials of total degree $\leq p$ on $\widehat{K}$, and let $\mathcal{Q}_{p}(\widehat{K})$ denote the set of polynomials of degree $\leq p$ in each variable $\left(\right.$ for $\left.n=1, \mathcal{P}_{p}(\widehat{I})=\mathcal{Q}_{p}(\widehat{I})\right)$. For an element $K_{i}=\mathcal{F}_{i}(\widehat{K})$, we define $\mathcal{R}_{p}\left(K_{i}\right)=$ $\left\{\widehat{v} \circ \mathcal{F}_{i}^{-1}: \hat{v} \in \mathcal{R}_{p}(\widehat{K})\right\}$ where $\mathcal{R}_{p}(\widehat{K})=\mathcal{P}_{p}(\widehat{K})$ if $\widehat{K}$ is an interval or triangle and $\mathcal{R}_{p}(\widehat{K})=\mathcal{Q}_{p}(\widehat{K})$ if $\widehat{K}$ is a quadrilateral. Let $\vec{p}_{N}=\left(p_{1}^{N}, \ldots, p_{M_{N}}^{N}\right)$ denote a degree vector. Then we define

$$
\begin{aligned}
V_{N}=V\left(\Omega, \vec{p}_{N}, \mathcal{T}_{N}\right)= & \left\{v \in C^{0}(\Omega),\left.v\right|_{\Omega_{i}} \in \mathcal{R}_{p_{i}}\left(K_{i}\right) \text { for } K_{i} \subset \mathcal{T}_{N}\right\} \\
& V_{N}^{0}=V_{N} \cap H_{0}^{1}(\Omega)
\end{aligned}
$$

If $p_{i}=p, i=1, \ldots, M_{N}$, then we simply write $p_{N}$ instead of $\vec{p}_{N}$.

\section{Basic mesh-degree design principles}

The underlying strategy in designing mesh-degree combinations for resolving boundary layers is most easily described in the one-dimensional case. Consider the one-dimensional problem

$$
\begin{gathered}
-d^{2} u_{d}^{\prime \prime}(x)+u_{d}(x)=f(x), x \in I=(-1,1), \\
u_{d}( \pm 1)=\alpha^{ \pm} .
\end{gathered}
$$

The above problem is singularly perturbed in the following sense. If $d$ is bounded away from zero, it is a second-order differential equation, but if $d=0$, (4) reduces to

$$
u_{0}(x)=f(x)
$$

which is of order 0 . Unless the boundary data (5) are compatible $\left(\alpha^{ \pm}=f( \pm 1)\right)$, the solution will contain boundary layer terms of the form

$$
u_{d}^{-}(x)=e^{-\frac{(x+1)}{d}}, u_{d}^{+}(x)=e^{-\frac{(1-x)}{d}} .
$$

In fact, the solution may be decomposed as follows [18].

Theorem 1 Let $M \in \mathbb{N}^{+}$be such that $f \in H^{4 M+2}(I)$. Then

$$
u_{d}(x)=u_{d}^{M}(x)+A_{d}^{M} u_{d}^{-}(x)+B_{d}^{M} u_{d}^{+}(x)
$$

where with $C=C\left(M, \alpha^{ \pm}, f\right)$ a constant independent of $d$, we have

$$
\left\|u_{d}^{M}\right\|_{H^{\ell}(I)}+\left|A_{d}^{M}\right|+\left|B_{d}^{M}\right| \leq C, \ell=0,1, \ldots, 2 M+2 .
$$


Theorem 1 shows that the finite element space $V_{N}$ we use should be able to approximate well the boundary layer terms $(7)$ and the smooth part $u_{d}^{M}(x)$. The strategy is to define two minimal finite element spaces $V_{N}^{B L}$ and $V_{N}^{S m}$ for each of these solution components, then choose $V_{N} \supset\left(V_{N}^{B L} \cup V_{N}^{S m}\right)$ (with appropriate boundary conditions being imposed).

For $V_{N}^{S m}$, since we only have to be able to approximate $u_{d}^{M}(x)$ which is a smooth function, the simplest space would be $\mathcal{P}_{p}(I)$. This is because if $u_{d}^{M}$ is entire analytic, the best asymptotic (i.e. as the polynomial degree $p \rightarrow \infty$ ) is to use only a single element. This yields super-exponential convergence in the energy norm for (4), i.e. the norm

$$
\|v\|_{E, d, S}:=\left(d^{2}|v|_{1, S}^{2}+\|v\|_{0, S}^{2}\right)^{1 / 2}
$$

where $S \equiv I \equiv(-1,1)$.

The space $V_{N}^{B L}$, on the other hand, must be designed to resolve the boundary layer ( 7 ) robustly, i.e. uniformly in $d$. This problem was considered in [18]. We have the following options:

(a) Patch-wise uniform $h$ version refinement

Suppose polynomials of degree $p \geq 1$ are used throughout. Let the mesh in the interior be quasiuniform with mesh-width $h$ and near the boundary with meshwidth $h_{0}$ (see Figure 3(a)). To maintain a robust $O(h)$ convergence in the energy norm, one finds easily that the mesh-width $h_{0}$ has to be used in a $O(p d|\ln d|)$ neighborhood of the boundary and that $h_{0}$ must be chosen to be $d h|\log h|$. This yields robust $O\left(N^{-1} \ln N\right)$ convergence (see [22], Lemma 2.53). Similar estimates can be obtained for the $L^{2}(I)$, the $H^{1}(I)$ and the $L^{\infty}(I)$-norm. This patch-wise uniform mesh design is sometimes also referred to as Shishkin mesh.

(b) Special refinement strategies for the $h$ version.

A better selection of $V_{N}^{B L}$ is based on judicious mesh refinement to suit the functions (7). Non-uniform meshes (e.g. [5], [9], [20]) can be used to yield robust $O\left(h^{p}\right)$ convergence. In Figure $3 \mathrm{~b}$ ), we have shown an example of an exponentially graded mesh for $p=1$, which was shown to be asymptotically optimal for functions (7) as $h \rightarrow 0$, in [21], [18]. This mesh is symmetric about $x=0$, and the mesh $\left\{x_{i}\right\}_{i=0}^{N}$ over $(-1,0)$ is given by

$$
x_{i}=\frac{1}{2} d(2 p+1) \ln \left(1-i \frac{C}{N}\right), i=0,1, \ldots, N,
$$

with $C=1-\exp (-2 / d(2 p+1))$. 

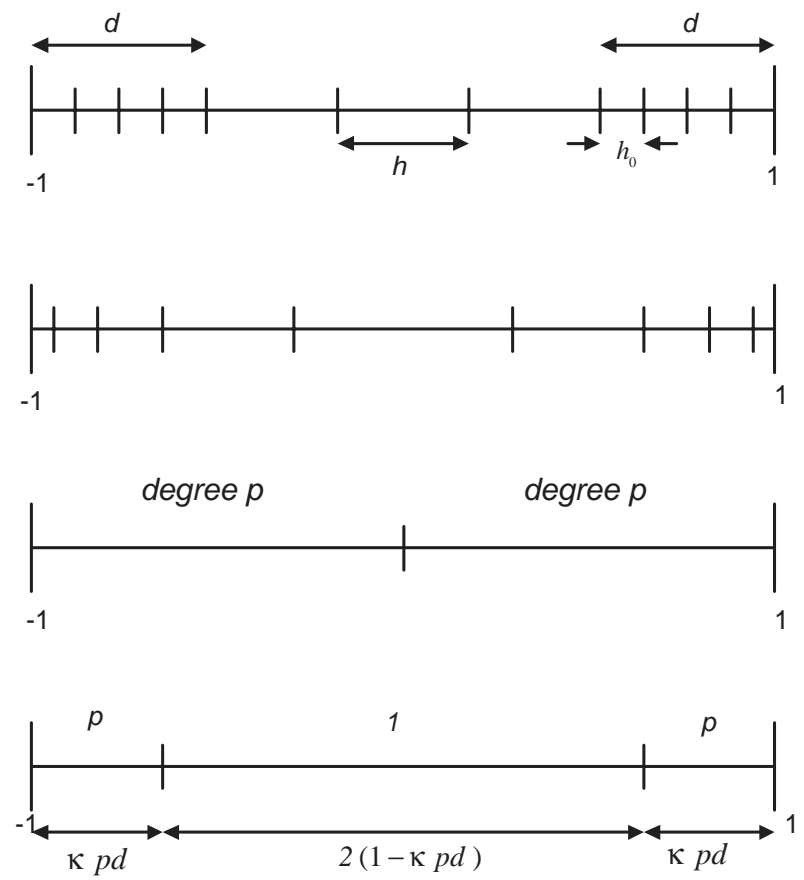

(a)

(b)

(c)

(d)

Figure 3: Choice of $V_{N}^{B L}$. a) Uniform end-point refinement. b) Exponential refinement. c) $p$ version on fixed uniform mesh. d) $h p$ version ( $p$ version on variable mesh)

We emphasize that these mesh families are asymptotically optimal, i.e. they yield the optimal convergence rate and also minimize the constant in the error estimate. Notice in (10) that as $p \rightarrow \infty$, the mesh grading in (10) becomes uniform, unlike the Shishkin type meshes described in (a) above. In our experiments, the mesh grading (10) gave better results than other meshes, such as the ones derived in [20] in terms of the number of unknowns, see [21] for details.

(c) p version refinement over a fixed (uniform) mesh.

It was shown in [18] that using $p$ refinement over a single element will, for any fixed $d$, give the best (superexponential) rate of convergence asymptotically as $p \rightarrow \infty$. Unfortunately, this rate is only visible when $p$ is very large $\left(p>C d^{-1}\right)$. For a practical choice of $p$ ( $p \leq 8$ in some commercial programs), the best rate that can be guaranteed, independent of $d$, is generally only $O\left(p^{-1}\right)$, which is very low. Hence this method is not recommended, unless a properly chosen mesh is used (see below).

(d) The hp version ( $p$ version on d-dependent mesh).

We have shown in [18] that proper combination of mesh refinement and increasing polynomial degree of the elements, i.e. the so-called $h p$-version FEM, can achieve exponential convergence for the boundary layers (1), uniformly in $\mathrm{d}$. The main result in [18] states that with the $h p$-FEM, for every boundary layer present,just one $O(p d)$-boundary layer element needs to be introduced at the respective boundary. In fact, for the model problem above, it is sufficient to use only 3 elements - an element 
of size $\kappa p d$ at each end, $0<\kappa<4 / e$, where degree $p$ is used, and an element of size $2(1-\kappa p d)$ in the middle. In this latter element, the degree can be as low as 1 , if only boundary layer functions are present in the solution, but the polynomial degree should be $p$ if smooth components are also present. (When $\kappa p d>1$ then the three elements collapse into one.) With this choice of space $V_{N}^{B L}$, it is possible to prove that the functions (7) are approximated exponentially in the energy norm as $p$ increases, i.e.

$$
\inf _{v \in V_{N}^{B L}}\left\|u_{d}^{ \pm}-v\right\|_{E, d} \leq C d^{1 / 2} \alpha^{p}
$$

where $\alpha<1$ and $C$ can be chosen independently of $p$ and $d$.

We see therefore from (a) - (d) above that for the problem (4) - (5), the best strategy is to choose $V_{N}^{S m}$ to be $\mathcal{P}_{p}(I)$ and to base $V_{N}^{B L}$ on the 3 element mesh in (d). If, in $V_{N}^{B L}$, we take the degree to be $p$ in the central element, then $V_{N}^{S m} \subset V_{N}^{B L}$, and we can take $V_{N}=V_{N}^{B L}$ (with appropriate modification for boundary conditions). We have the following theorem.

Theorem 2 [18] For $0<\kappa<4 / e, 0<d \leq 1$, let $\left\{\mathcal{T}_{p}\right\}$ be a sequence of meshes defined on $I=[-1,1]$, with

$$
\begin{aligned}
& \left\{\mathcal{T}_{p}\right\}=\{-1,-1+\kappa p d, 1-\kappa p d, 1\} \text { if } \kappa p d<1 \\
& \left\{\mathcal{T}_{p}\right\}=\{-1,1\} \text { if } \kappa p d \geq 1 .
\end{aligned}
$$

Let $\vec{p}=\{p, p, p\}$ and define $V_{p}=V\left(I, \vec{p}, \mathcal{T}_{p}\right)$. Let $u_{d, p}$ be the finite element approximation to (4) - (5) (with the test space being $V_{p} \cap H_{0}^{1}(I)$ ), and assume $f \in \mathcal{P}_{n}(I)$. Then for $p \geq n$,

$$
\left\|u_{d}-u_{d, p}\right\|_{E, d} \leq C d^{1 / 2} \alpha^{p}
$$

where $\alpha<1$ and $C$ are constants independent of $p$ and $d$.

Remark 3 The conditions $f \in \mathcal{P}_{n}(I), p \geq n$ are technical assumptions that ensure the exponential convergence of the smooth part $u_{d}^{M}$. These technical assumptions can be removed, see [12], provided $f$ is analytic in $[-1,1]$. If $f$ is not smooth enough, the rate (1D) will be $O\left(p^{-k}\right)$, where $k$ is determined by the smoothness of $u_{d}^{M}$.

Remark 4 Note that we are not using the full power of the hp version, since we don't increase the number of elements but only change the size of the elements as p increases. For practical purposes, our (D-D) computational experiments in Section 3 indicate that it is possible to avoid the inconvenience of changing the mesh with every $p$ by using the fixed mesh $\mathcal{T}_{p_{\max }}$ for all $p, 1 \leq p \leq p_{\max }$ used in a given hp-FE implementation.

Let us show the results of some computations performed for problem (4) - (5), with $f=1, \alpha^{ \pm}=0$. The exact solution is given by

$$
u_{d}(x)=1-\frac{e^{-\frac{(x+1)}{d}}}{e^{\frac{1}{d}}+e^{-\frac{1}{d}}}-\frac{e^{-\frac{(1-x)}{d}}}{e^{\frac{1}{d}}+e^{-\frac{1}{d}}},
$$


which is clearly of the form (8). In Figure 4, we test different values of $\kappa$ for the $h p$ method, and find $\kappa=1$ is the best. In Figures $5-7$ we show the different methods above for various values of $d$ (method (d) uses $\kappa=1)$. Clearly, the $h p$ version gives the best, most robust results.

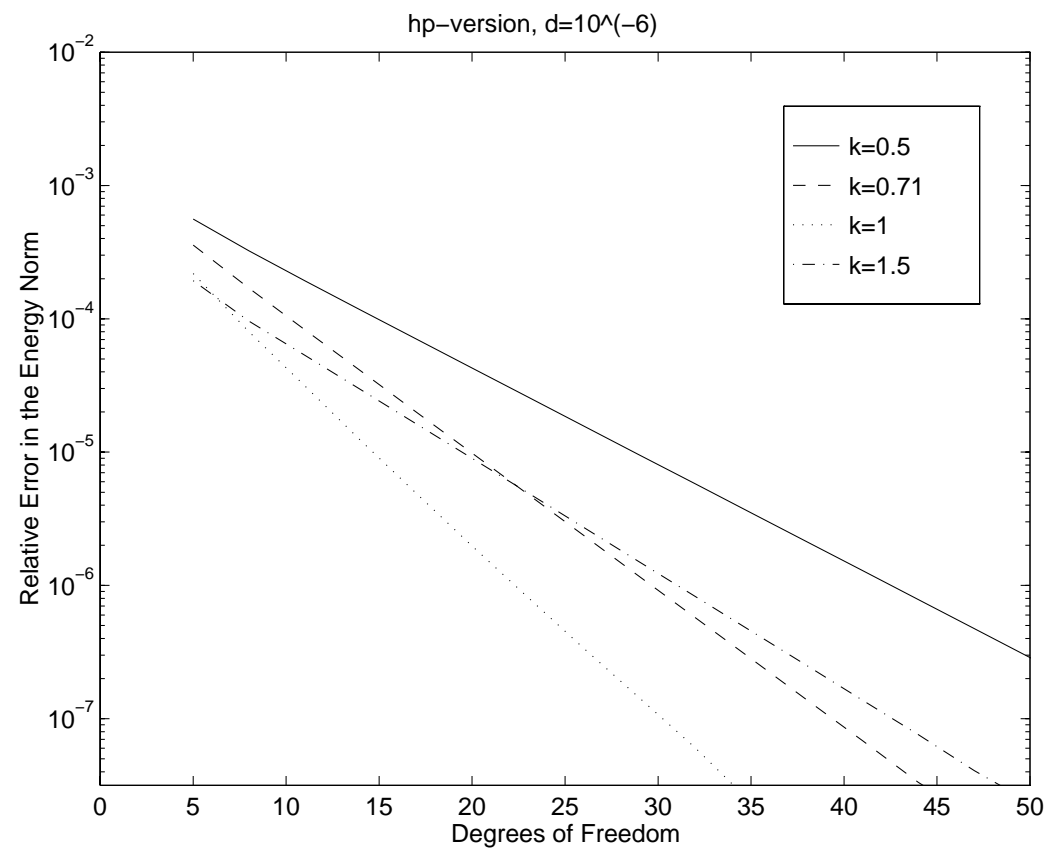

Figure 4: The dependence on the parameter $\kappa$.

\section{Problems over smooth domains}

The principles from Section 2 are immediately applicable to the mesh-degree design for higher-dimensional problems with boundary layers, provided the solution has no other singular components (such as corner singularities). This is in general the case when the domain $\Omega$ is smooth (or when appropriate symmetric/antisymmetric boundary conditions are applied, as in the example in Section 1). The boundary layers now show up as functions like (1) (in 3-D, $\tau$ may be a function of $x, y$ and $z$ ).

Let us define boundary-fitted coordinates (Figure 8). Given a smooth domain $\Omega \subset \mathbb{R}^{2}$, let

$$
\Omega_{0}=\left\{z-\rho \vec{n}_{z}: z \in \partial \Omega, 0<\rho<\rho_{0} \leq \text { minimum radius of curvature of } \partial \Omega\right\}
$$

where $\vec{n}_{z}$ denotes the outward unit normal at $z \in \partial \Omega$. The boundary-fitted coordinates $(\rho, \theta)$ are defined by the correspondence

$$
(\rho, \theta) \rightarrow z-\rho \vec{n}_{z}=\left(X(\theta)-\rho Y^{\prime}(\theta), Y(\theta)+\rho X^{\prime}(\theta)\right),
$$

where $z=z(\theta)=(X(\theta), Y(\theta))$ denotes the parametric representation of $\partial \Omega$ in terms of the arclength $\theta$. 


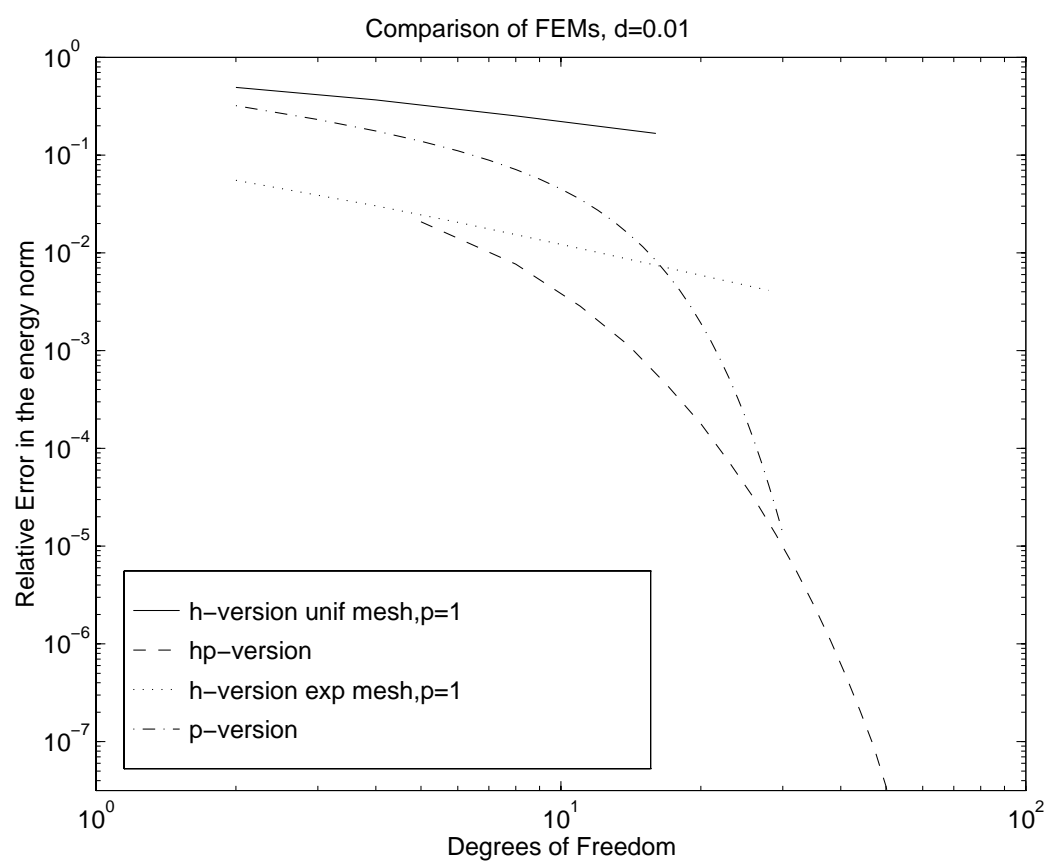

Figure 5: Comparison of various methods, $d=10^{-2}$.

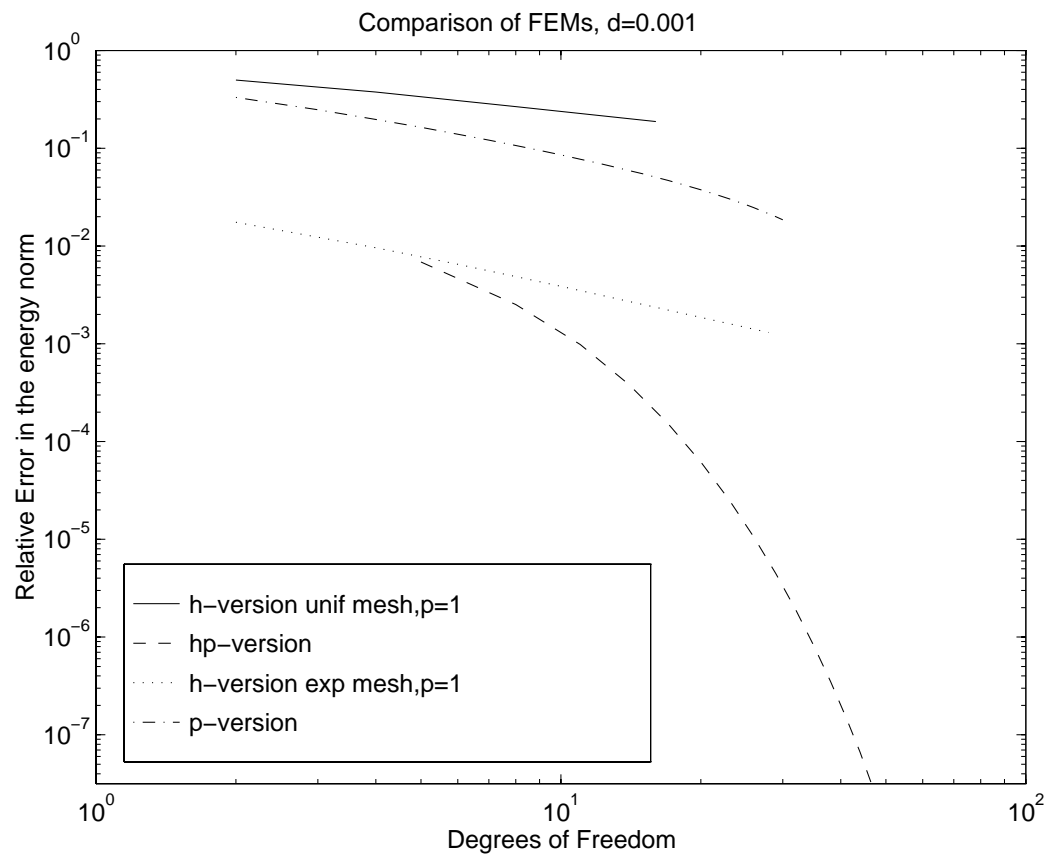

Figure 6: Comparison of various methods, $d=10^{-3}$. 


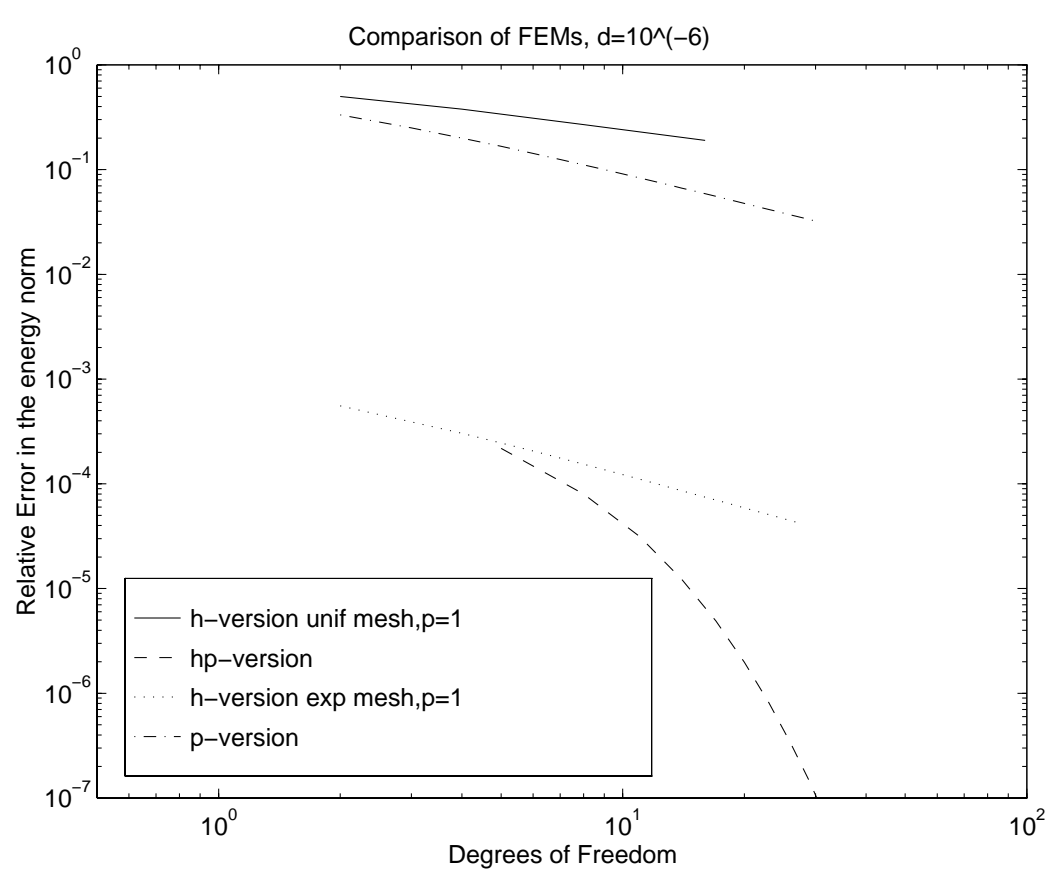

Figure 7: Comparison of various methods, $d=10^{-6}$.

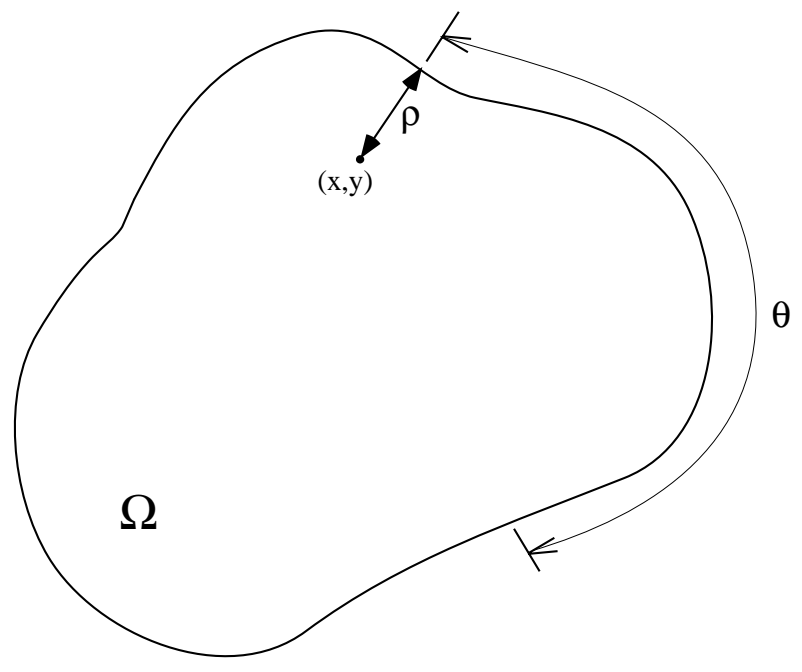

Figure 8: Boundary fitted coordinates in $\Omega_{0}$. 
For many problems of interest, it turns out that when the functions $\xi$ in (1) are expressed in terms of $(\rho, \theta)$, they have the form

$$
\xi(\rho, \theta)=\sum_{i=0}^{q} \tau_{i}(\theta) \rho^{i} e^{-\alpha \rho / d},
$$

where $\tau_{i}(\theta)$ are smooth. We consider two such problems in this section - the heat transfer problem and the Reissner-Mindlin plate problem. (Other problems, like the Naghdi shell model are commented on in Section 5.)

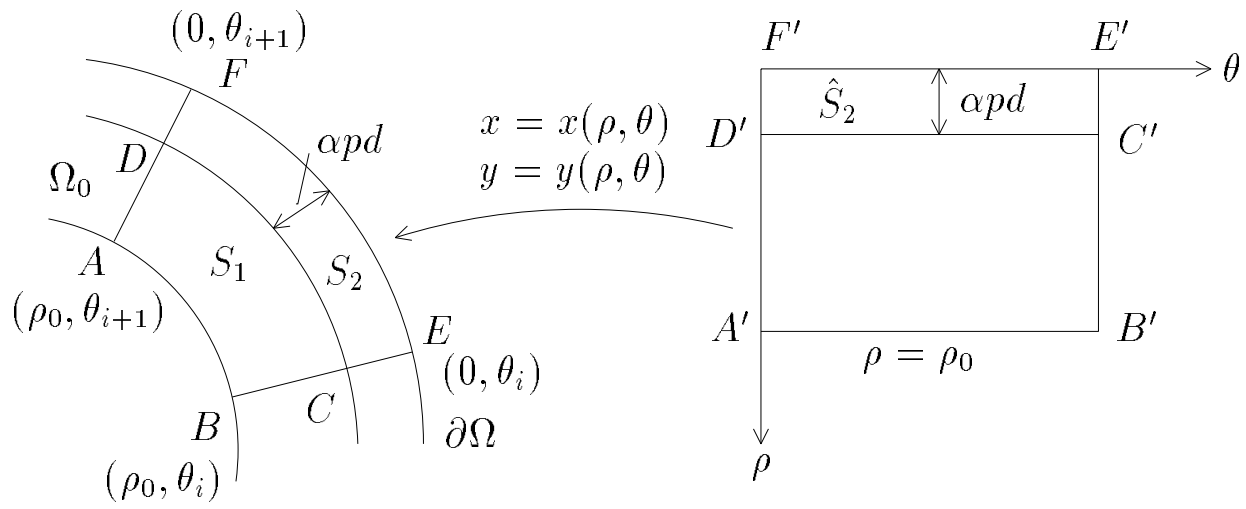

Figure 9: Boundary-fitted elements in $\Omega_{0}$.

If we now look at the components of (13), we see that the boundary layer effect is still only a one-dimensional one, in the direction of $\rho$ (the functions $\tau_{i}(\theta)$ being smooth). Hence we may define boundary-fitted elements (as shown in Figure 9) on $\Omega_{0}$. We do this by dividing $\partial \Omega$ into subintervals $\left(\theta_{i}, \theta_{i+1}\right), 1 \leq i \leq m-1, \theta \in \partial \Omega$ and drawing the inward normal at $\theta_{i}, 1 \leq i \leq m$, of length $\rho_{0}$. Then the points $\left(\rho_{0}, \theta_{i}\right)$ are connected by the curve $\rho=\rho_{0}$. Each curvilinear quadrilateral $S=A B E F$ is then further subdivided into two elements $S_{1}$ and $S_{2}$ by the curve $\rho=\kappa p d$, according to the prescription in the previous section. Looking at $A B E F$ in the $(\rho, \theta)$ coordinates then gives two rectangular elements $\widehat{S}_{1}=A^{\prime} B^{\prime} C^{\prime} D^{\prime}$ and $\widehat{S}_{2}=D^{\prime} C^{\prime} E^{\prime} F^{\prime}$ as shown in Figure 9. The local polynomial space on $S_{i}, i=1,2$ is then defined (using the notation $v(x, y)=\hat{v}(\rho, \theta)$ for $(x, y)=$ $(x(\rho, \theta), y(\rho, \theta)))$ by

$$
\mathcal{Q}_{p}\left(S_{i}\right)=\left\{v(x, y): \hat{v}(\rho, \theta) \in \mathcal{Q}_{p}\left(\widehat{S}_{i}\right)\right\}
$$

Note that the basis functions we use are polynomials in $(\rho, \theta)$ instead of in $(x, y)$.

Consider the local approximation of (13) over the space

$$
V_{p}(S)=\left\{v \in C^{0}(S):\left.v\right|_{S_{i}} \in \mathcal{Q}_{p}\left(S_{i}\right)\right\}
$$

The function $\tau_{i}$ being smooth, is approximated exponentially by a piecewise polynomial $\tau_{i}^{p}(\theta)$ of degree $p$. The function $\exp (-\alpha \rho / d)$ is approximated at an exponential rate by a piecewise polynomial $v(\rho)$, of degree $p-q$, as in (11). Then, for $q$ fixed, $p$ large enough, 
we obtain, by a simple tensor product argument (see [21]), that

$$
\left\|\xi(\rho, \theta)-\sum_{i=0}^{q} \tau_{i}^{p}(\theta) \rho^{i} v(\rho)\right\|_{E, d, S} \leq C d^{1 / 2} \alpha^{p}
$$

so that the local approximation in the energy norm is the same as that in the onedimensional case.

Remark 5 So far, we considered only boundary-fitted meshes. We expect that analogous results are also valid for more general, properly refined triangulations at the boundary as used in various codes.

\subsection{The heat transfer problem}

We consider the problem

$$
\begin{gathered}
-d^{2} \Delta u_{d}+u_{d}=f(x, y) \text { in } \Omega \\
u_{d}=0 \text { on } \partial \Omega,
\end{gathered}
$$

where $d \in(0,1]$. This is the two-dimensional analog of (4) - (5) and can be used to model, for example, heat conduction in a thin domain (see e.g. [25], [24]) where $d$ is a thickness parameter.

Once again, this problem is singularly perturbed since (15) reduces to the analog of (6) for $d=0$. Except for special $f$, the solution will contain boundary layer terms. The following decomposition was established in [21] (see also [7], where other decompositions may be found). The function $\chi$ is a $C^{\infty}([0, \infty))$ cut-off function satisfying

$$
\chi(r)=\left\{\begin{array}{l}
1 \text { for } 0 \leq r \leq \rho_{0} / 3 \\
0 \text { for } r \geq 2 \rho_{0} / 3
\end{array}\right.
$$

and $\left|\chi^{(m)}(r)\right| \leq C\left(\rho_{0}, m\right), m=0,1, \ldots$.

Theorem 6 [21] Let $u_{d}$ be the solution of (15)-(16), and assume $f \in H^{4 M+2}(\Omega)$ for some $M \in \mathbb{N}$. Then

$$
u_{d}=u_{d}^{M}+\chi u_{d, B L}^{M}+r_{d}^{M}
$$

where

$$
\begin{gathered}
u_{d}^{M}(x, y)=\sum_{i=0}^{M} d^{2 i} \Delta^{(i)} f(x, y), \\
u_{d, B L}^{M}=\sum_{i=0}^{M} d^{i} \sum_{k=0}^{i} \tau_{k i}(\theta) \frac{\rho^{k}}{d^{k}} e^{-\rho / d}=\sum_{i=0}^{M} \tau_{i}(\theta) \rho^{i} e^{-\rho / d}
\end{gathered}
$$

and

$$
\left\|r_{d}^{M}\right\|_{k, \Omega} \leq C d^{M+3 / 2-k}, 0 \leq k \leq M+3 / 2
$$

with $C$ a constant independent of $d$ and $\tau_{i k}(\theta)$ smooth and independent of $d$. 
Remark 7 If $f \in \mathcal{P}_{n}(\Omega)$, then we can take $M$ arbitrarily high in (17) - (20). For any $M$, however, the summations in (18) - (19) will now only be from $i=0$ to $n$. Hence, for any $M, u_{d, B L}^{M}$ reduces to $\xi(\rho, \theta)$ given by (13) $(\alpha=1)$, where $n$ is fixed. In contrast, when we have a general $f$, then reducing the size of the remainder in (DO) by taking $M$ large can only be done at the expense of adding more terms to (19). The situation is analogous to that discussed in Remark 3.

We now define a sequence of meshes $\left\{\mathcal{T}_{N}\right\} \equiv\left\{\mathcal{T}_{p}\right\}$ on $\Omega$, such that $\Omega_{0}$ is divided into two layers of elements as shown in Figure 9, and the mesh in $\Omega \backslash \Omega_{0}$ is compatible with the mesh on $\Omega_{0}$. Then defining $V_{N}^{0} \equiv V_{p}^{0}=V\left(\Omega, \vec{p}, \mathcal{T}_{p}\right) \cap H_{0}^{1}(\Omega)$ as in $(2)-(3)$, we see that the boundary-fitted elements in $\Omega_{0}$ will approximate $u_{d, B L}^{M}$ exponentially as $p \rightarrow \infty$. Moreover, since $u_{d}^{M}$ and $r_{d}^{M}$ are smooth, they will be approximated very well by $V_{p}$ as $p \rightarrow \infty$. When $f \in \mathcal{P}_{n}(\Omega)$ as in Remark 7 , and $p \geq n$, we will have at least arbitrarily high algebraic convergence.

More precisely, let $u_{d, p} \in V_{p}^{0}$ be the finite element solution of

$$
B_{d}\left(u_{d, p}, v\right)=\int_{\Omega} \nabla u_{d, p} \cdot \nabla v d x=\int_{\Omega} f v d x \forall v \in V_{p}^{0} .
$$

The following theorem is established in [21].

Theorem 8 Let $u_{d}, u_{d, p}$ be the solutions to (15) - (16) and (21) respectively. Let $f \in$ $\mathcal{P}_{n}(\Omega)$. Then for any $s>0$, there exists a constant $C(s)$ independent of $p, d$ such that

$$
\left\|u_{d}-u_{d, p}\right\|_{E, d, \Omega} \leq C(s) p^{-s}
$$

for $p \geq n$.

Remark 9 The rate (D2) is practically exponential, as will be seen from the experiments below. For the case of general $f$, (DD) will again hold, but possibly only for a finite range of $s$.

Let us now present some numerical results. We consider (15) - (16) over the unit disk with $f \equiv 1$. The exact solution in polar coordinates is

$$
u_{d}(r, \theta)=u_{d}(r)=1-\frac{I_{0}(r / d)}{I_{0}(1 / d)}
$$

where $I_{0}(z)$ is the modified Bessel function of order zero. We have plotted $u_{d}(r)$ for $0 \leq r \leq 1$ in Figure 10 to illustrate the boundary layer nature.

We compare two finite element schemes, each with 8 elements, but on the two different meshes shown in Figure 11. The first is a uniform mesh, while in the second, there is an $O(p d)$ layer of elements along the boundary. Letting $p$ increase on the second mesh corresponds to using the specially designed spaces $V_{p}$ on which Theorem 8 is based, while on the first mesh, we just get the usual $p$ version.

We performed experiments for various values of $d$ using the code STRESS CHECK. For ease of computation, we took the thickness of the outer layer in the second mesh to 


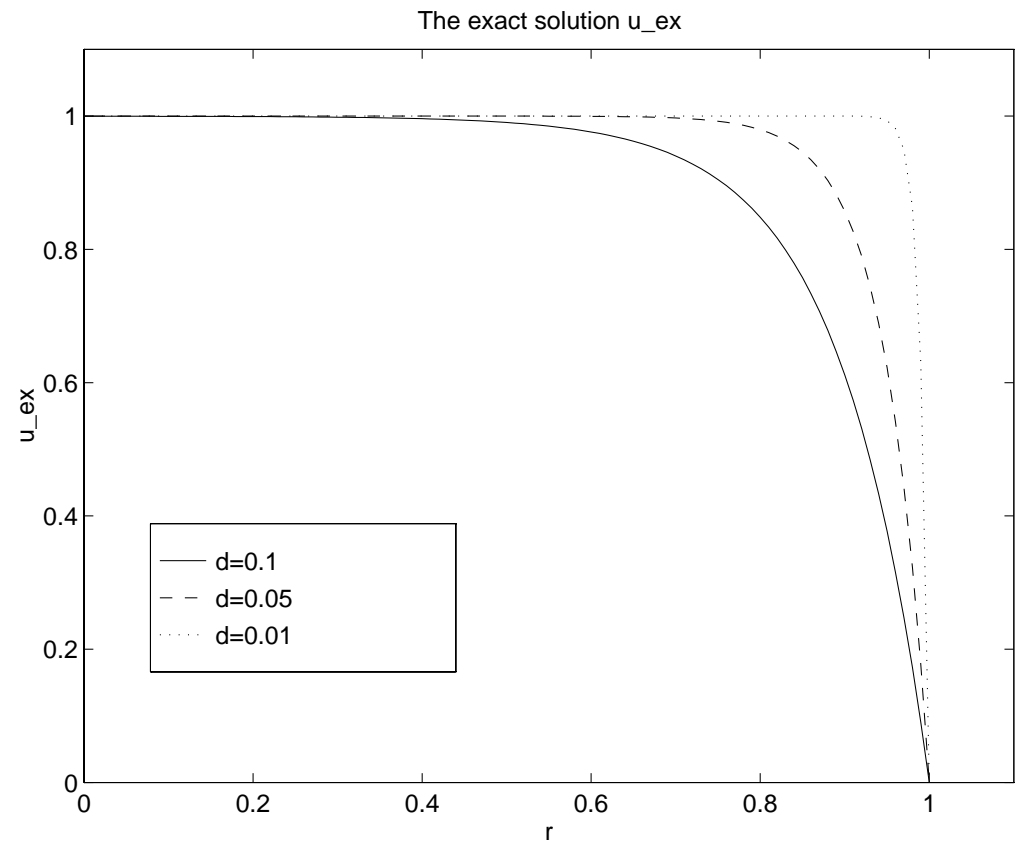

Figure 10: The exact solution $u_{d}(r)$.

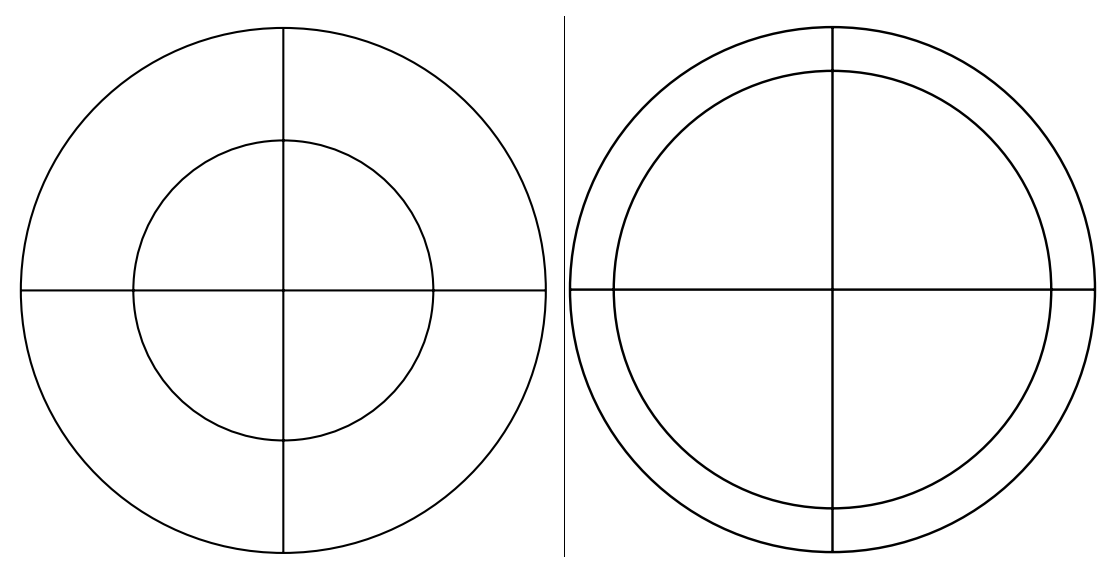

Figure 11: The meshes for schemes 1 and 2. 

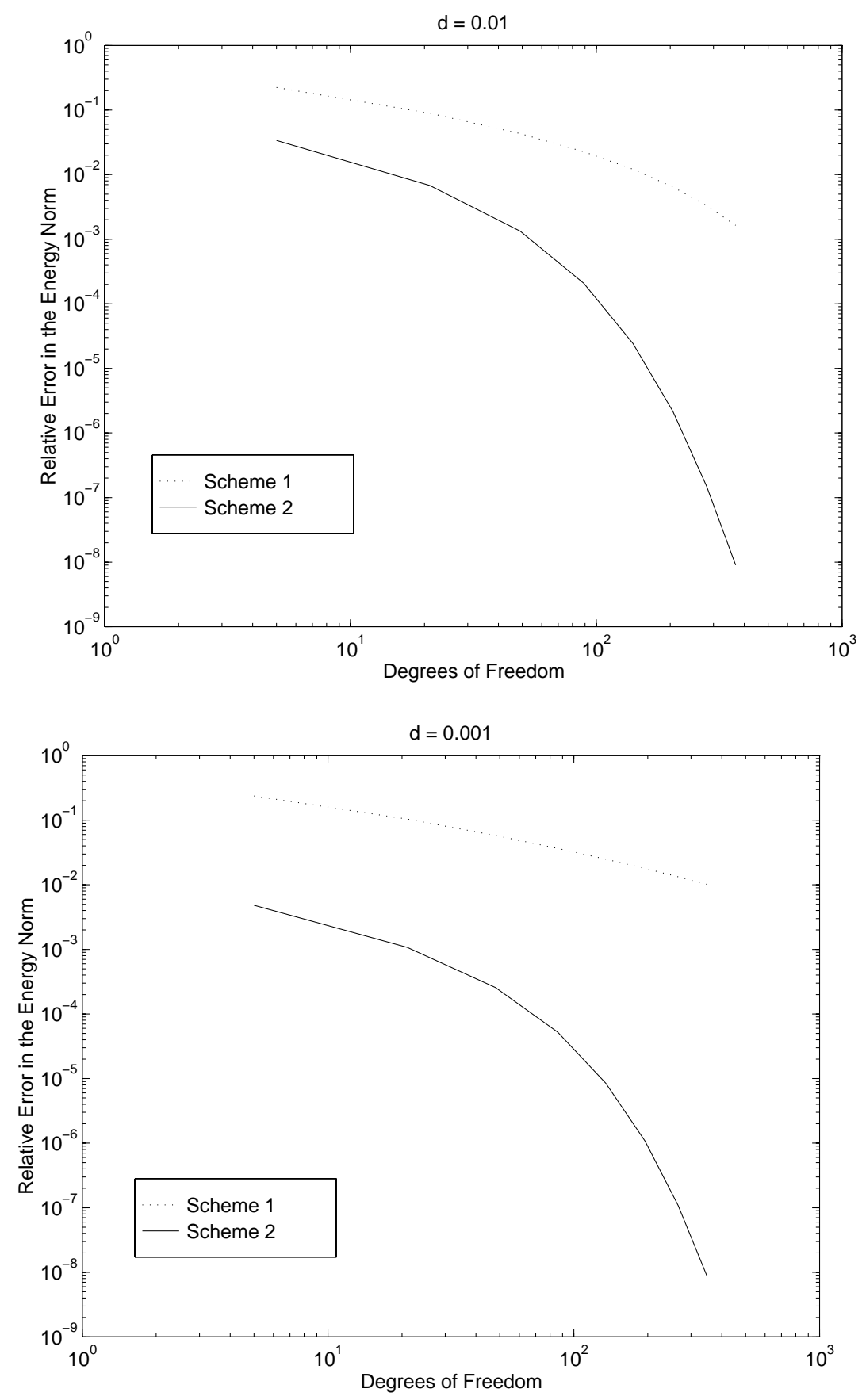

Figure 12: Energy norm comparison for the two schemes: $d=0.01, d=0.001$. 
be $p_{\max } d$ (rather than $p d$ ), for all $p=1,2, \ldots, 8=p_{\max }$. In Figure 12 we plot the relative error in the energy norm versus the number of degrees of freedom, $N$. It is seen that the first scheme deteriorates for small $d$, while the second is robust, and demonstrates the expected exponential rate.

We also consider the relative error in the values of $\frac{d u_{d}}{d r}$ at the point $(r, \theta)=(1,0)$ on the boundary of $\Omega$. It is observed from Figure 13 that now the deterioration of the first scheme is even more pronounced (as can be expected from the fact that for $\frac{d u_{d}}{d r}$, the boundary layer in (23) gets multiplied by a factor $d^{-1}$ ). Hence, it is even more essential to correctly design the mesh when the fluxes are of interest.

\subsection{The Reissner-Mindlin plate problem}

Let us now consider the bending of a homogeneous isotropic plate of thickness $d$, occupying the region $R=\Omega \times(d / 2, d / 2)$ (where $\partial \Omega$ is again smooth), under a normal load density per unit area given by $g d^{3}$. The equations of equilibrium for $\vec{\phi}_{d}$, the rotation, and $w_{d}$ the transverse displacement, are

$$
\begin{gathered}
-\frac{D}{2}\left\{(1-\nu) \Delta \vec{\phi}_{d}+(1+\nu) \nabla \nabla \cdot \vec{\phi}_{d}\right\}-\tilde{\kappa} \mu d^{-2}\left(\nabla w_{d}-\vec{\phi}_{d}\right)=0 \\
-\tilde{\kappa} \mu d^{-2} \nabla \cdot\left(\nabla w_{d}-\vec{\phi}_{d}\right)=g .
\end{gathered}
$$

Here, $\nu$ is the Poisson ratio, $E$ the Young's modulus, $D=E /\left(12\left(1-\nu^{2}\right)\right), \mu=E /(12(1+$ $\nu)$ ), and $\tilde{\kappa}$ is the shear correction factor.

The elliptic system (24)-(25) is once again singularly perturbed, as can be seen by multiplying (24)-(25) by $d^{2}$. For $d>0$, this system contains derivatives of order 2 in $\vec{\phi}_{d}$ and 2 in $w_{d}$. However, for $d=0$, we only get derivatives of order 1 in $\vec{\phi}_{d}$ and 2 in $w_{d}$. Hence, there will be a boundary layer in $\vec{\phi}_{d}$ (but there is not one in $w_{d}$ ). The strongest layers occur when either free or soft simple support boundary conditions are imposed - here, we restrict our attention to the latter. The following theorem, analogous to Theorems 1, 6 gives the decomposition for the solution.

Theorem 10 [1] Let $u_{d}=\left(\vec{\phi}_{d}, w_{d}\right)$ be the solution of (2/) - (25) and assume $g \in$ $H^{3 M-4}(\Omega)$ for some $M \in \mathbb{N}$. Then

$$
u_{d}=u_{0}+d u_{d}^{M}+d \chi u_{d, B L}^{M}+r_{d}^{M}
$$

where

$$
u_{0}=\left(\operatorname{grad} w_{0}, w_{0}\right)
$$

solves the limiting Kirchhoff problem,

$$
u_{d}^{M}=\left(\vec{\phi}_{d}^{M}, w_{d}^{M}\right)=\sum_{i=1}^{M} d^{i-1}\left(\vec{\phi}_{i}, w_{i}\right)
$$



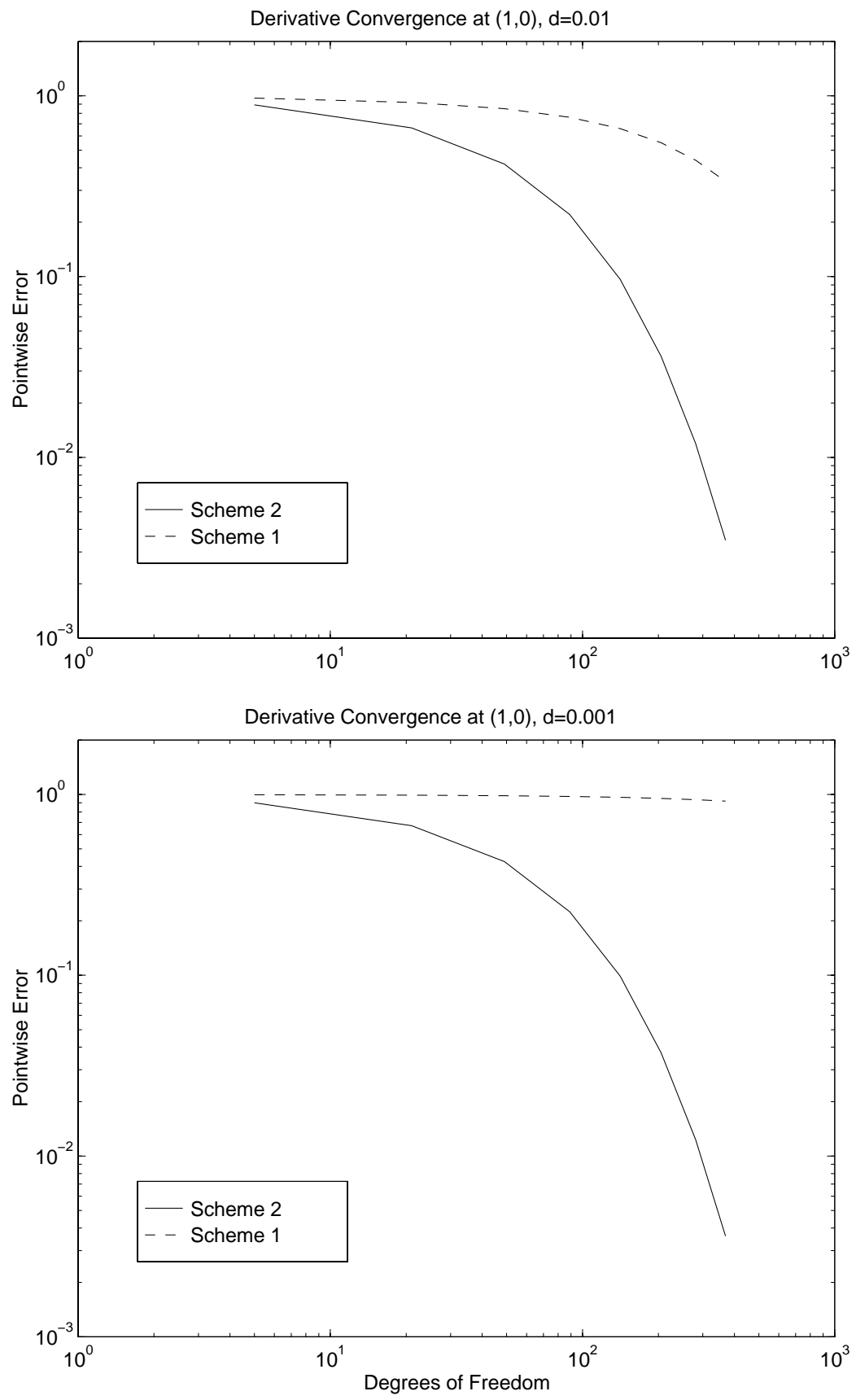

Figure 13: Flux comparison for the two schemes 
with $\left\|\phi_{i}\right\|_{k},\left\|w_{i}\right\|_{k+1} \leq C$ uniformly in $d$ for $0 \leq k \leq M$,

$$
u_{d, B L}^{M}=\left(\vec{\phi}_{d, B L}^{M}, 0\right)=\left(\sum_{i=1}^{M} d^{i} \vec{\Pi}_{i}(\rho / d, \theta) e^{-\sqrt{12 \tilde{\kappa}} \rho / d}, 0\right)
$$

where $\vec{\Pi}_{i}(r, \theta)$ are polynomials of degree $i$ in $r$ with smooth L-periodic (in $\theta$ ) coefficients, and the remainder $r_{d}^{M}=\left(\vec{\phi}_{d}^{M}, w_{d}^{M}\right)$ satisfies

$$
\begin{gathered}
\left\|\vec{\phi}_{d}^{M}\right\|_{k} \leq C d^{M+3 / 2-k}, 0 \leq k \leq M \\
\left\|w_{d}^{M}\right\|_{k} \leq C d^{M+1}, 0 \leq k \leq M .
\end{gathered}
$$

See [1] for more precise estimates.

Theorem 10 shows that we have a boundary layer term only in $\vec{\phi}_{d}$, with a coefficient which is of size $O(d)$, rather than $O(1)$ as in the heat problem. Since this layer is weaker, we expect there to be some improvement in its numerical approximation (this is seen in the results below). For other boundary conditions, such as hard clamped conditions, the layer is even weaker, since the coefficient is $O\left(d^{2}\right)$ now.

The numerical approximation of (26) is now complicated by another factor, however - that of locking. As $d \rightarrow 0$ in (26), we see that $u_{d} \rightarrow u_{0}$ satisfying (27). It turns out that we are now forced to find a finite element solution $\left(\vec{\phi}_{d, N}, w_{d, N}\right)$ which also takes the form $\left(\operatorname{grad} w_{0, N}, w_{0, N}\right)$ in the limit as $d \rightarrow 0$. Often, the finite element spaces may not contain enough elements satisfying this limiting constraint, with the result that the approximations deteriorate as $d \rightarrow 0$. This is called locking, and the spaces (and method) must be carefully tailored to avoid it. See e.g. [19], [15], [17], [4].

Due to the possible presence of locking, it is cumbersome to state exact conditions under which an analog of Theorem 8 will hold for plates (see [19]). We therefore only note here that a similar strategy of constructing a boundary layer mesh in $\Omega_{0}$ will be sufficient to resolve the boundary layer from an approximation theory point of view. In fact, as our experiments below indicate, this boundary layer refinement is necessary if pointwise convergence of the stresses is of interest. (Evidence to this effect was already provided in the introduction for a plate with periodic boundary conditions.) Also, use of high $p$ effectively controls locking, as shown in [15], [17].

We consider here a circular plate with the two meshes shown in Figure 11. For the case of soft simple support, an exact solution in the case $g=\cos (\theta)$ was given in [2] in polar coordinates : $u_{d}=\left(\phi_{r}, \phi_{\theta}, w\right)$ where

$$
\begin{aligned}
\phi_{r} & =\left(4 r^{3} /(45 D)+3 a r^{2}+b-c \lambda^{-1} d^{2}+r^{-1} \lambda^{-1} f d^{2} I_{1}(\tilde{\kappa} r / d)\right) \cos \theta \\
\phi_{\theta} & =\left(-r^{3} /(45 D)-a r^{2}-b+c \lambda^{-1} d^{2}-d \tilde{\kappa} \lambda^{-1} f I_{1}^{\prime}(\tilde{\kappa} r / d)\right) \sin \theta \\
w & =\left(r^{4} /(45 D)-\lambda^{-1} d^{2} r^{2} / 3+a\left(r^{3}-8 D \lambda^{-1} r d^{2}\right)+b r-c \lambda^{-1} r d^{2}\right) \cos \theta .
\end{aligned}
$$

Here, $\lambda=E \tilde{\kappa} /(2(1+\nu)), I_{1}(z)$ is the modified Bessel function of order 1 , and the constants $a, b, c, f$ are given explicitly in [2]. 
In Figure 14, we show the energy norm error for the two schemes, for $d=0.1$ and $d=0.01$. Even though some deterioration is seen for $d=0.01$, the difference is not as significant as was observed in Figure 12 for the heat transfer problem. This is due to the extra ' $d$ ' factor in front of the boundary layer term in (26) compared to (17). However, as is seen from Figure 15, if we look at the shear stress along the line $0.99 \leq r \leq 1$, near the boundary, then the fact that this involves a derivative causes the difference between the two schemes to become more pronounced.
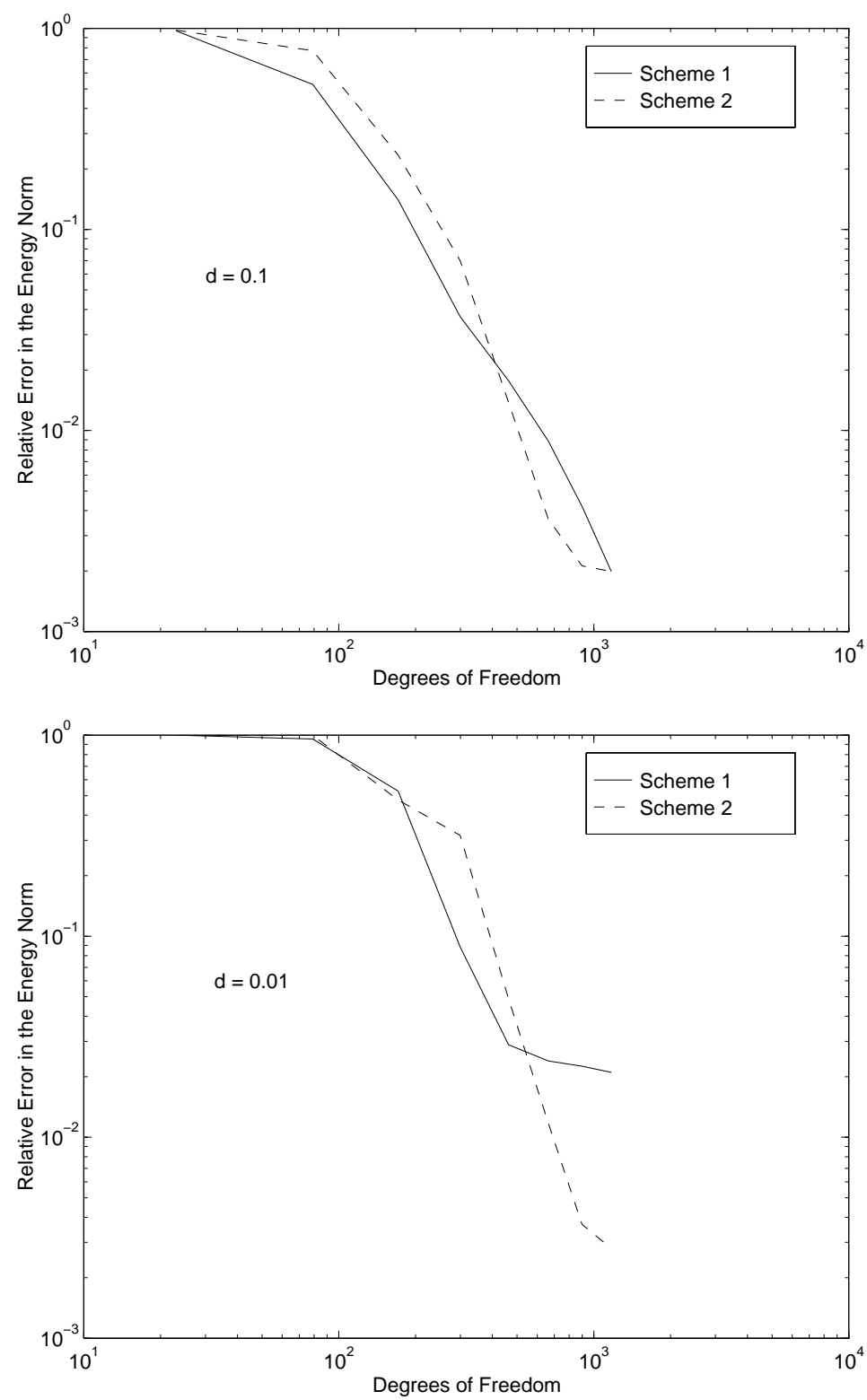

Figure 14: Energy norm comparison for the two schemes: $d=0.1, d=0.01$. 

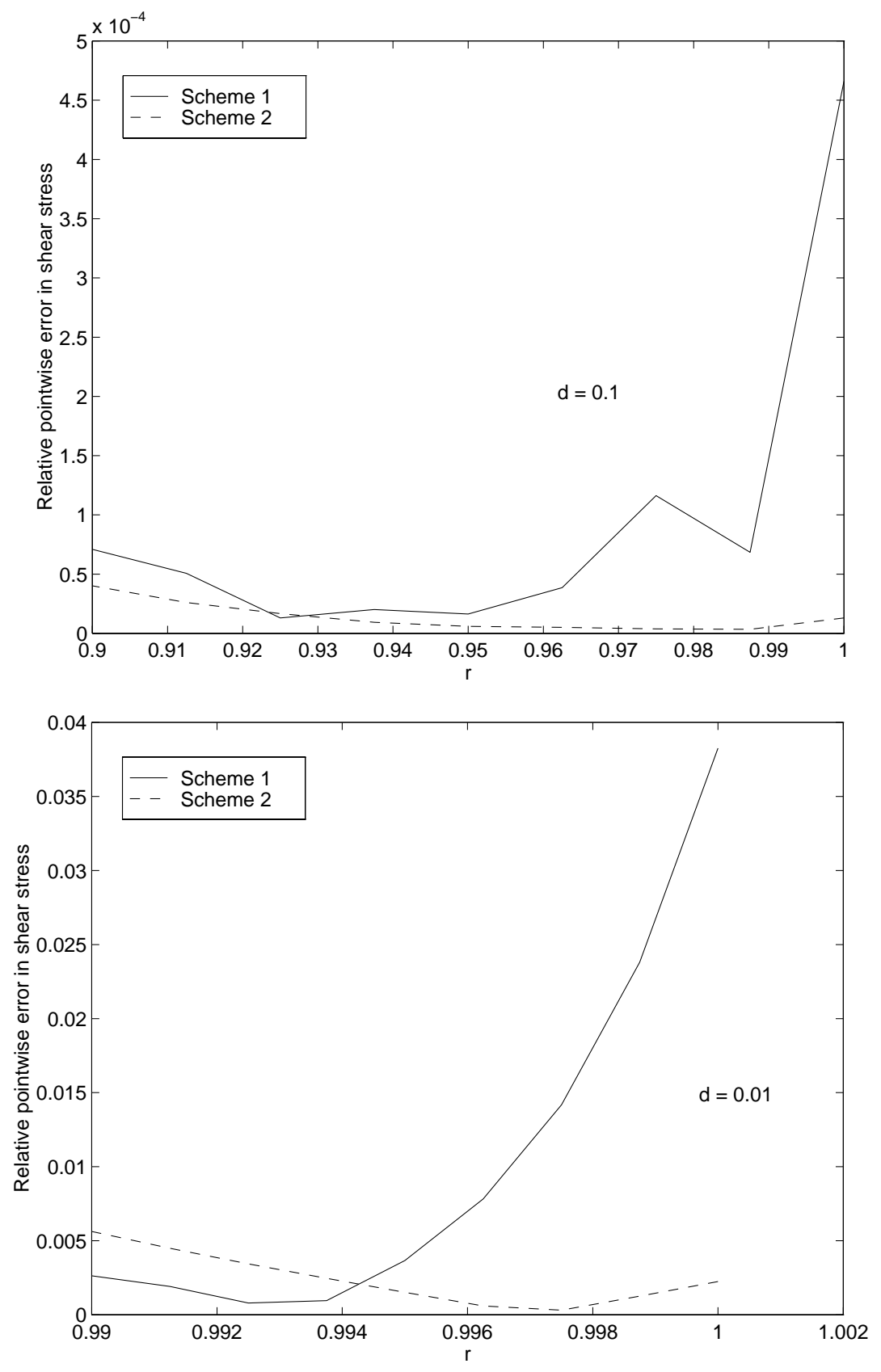

Figure 15: Comparison of relative error in point-wise shear stress for $d=0.1, d=0.01$. 


\section{Problems over non-smooth domains}

It is well-known that for elliptic problems over non-smooth domains, singular components are introduced in the solution in neighborhoods of the corners. These singularities behave like $f(r) \sim r^{\alpha}(\log r)^{\beta}$ where $r$ is the distance from the corner. For singularly perturbed problems, the behavior of the singularities and their interaction with boundary layers is a complicated affair. In general, the solution for finite $d$ will now contain boundary layers, corner singularities of the form $f(r / d)$, and, moreover, possible corner singularities of the limit problem $(d=0)$ of the form $r^{\alpha}(\log r)^{\beta}$. Relatively few mathematical decompositions similar to the ones in Theorems 1, 6, 10, are available at this point for such problems, with the case of the heat transfer problem being, perhaps, the most well understood ([10], [11]).

In this section, we consider a model heat transfer problem over a square for which an explicit decomposition is known. We show how the mesh design of the previous section should be modified in this case, to capture the corner singularities as well. We present theoretical and computational results from [21] proving uniform spectral convergence. Guided by this example, we show how problems over more general polygons should be treated.

\subsection{The heat transfer problem over a square}

We consider the problem (15) - (16) posed over the unit square $\Omega=(0,1)^{2}$. The solution can be decomposed using the results in [10], [6]. The case $f \equiv 1$ is the simplest, and is the one we consider here, but similar theorems will hold for $f \in \mathcal{P}_{n}(\Omega)$ as well.

Let us define the corner singularity functions $\tilde{Z}(\xi, \eta)$ to be the solutions of

$$
\begin{gathered}
-\left(\tilde{Z}_{\xi \xi}+\tilde{Z}_{\eta \eta}\right)+\tilde{Z}=0 \text { in } Q_{1}=(0, \infty) \times(0, \infty) \\
\tilde{Z}(0, \eta)=e^{-\eta}, \tilde{Z}(\xi, 0)=e^{-\xi}, \xi, \eta \in \mathbb{R}^{+} \\
\tilde{Z}(\xi, \eta) \rightarrow 0 \text { as }|\xi|+|\eta| \rightarrow \infty .
\end{gathered}
$$

These functions are used in Theorem 11 below.

Theorem 11 [10] Let $u_{d}$ be the solution of (15) - (16) with $f \equiv 1$ and $\Omega=(0,1)^{2}$. Then

$$
u_{d}=u_{S m}+u_{d, B L}+u_{d, C o}+r_{d}
$$

where $u_{S m}=1$, the boundary layer function $u_{d, B L}$ is given by

$$
u_{d, B L}=e^{\frac{-x}{d}}+e^{\frac{-y}{d}}+e^{\frac{-(1-x)}{d}}+e^{\frac{-(1-y)}{d}},
$$

the corner layer function $u_{d, C o}$ has the structure

$$
u_{d, C_{0}}=Z(x, y)+Z(x, 1-y)+Z(1-x, y)+Z(1-x, 1-y)
$$

with $Z(x, y)=\tilde{Z}(x / d, y / d), \tilde{Z}(\xi, \eta)$ defined as in (28) - (30), and the remainder $r_{d}$ satisfies

$$
\left\|r_{d}\right\|_{m, \Omega} \leq C_{m} e^{-a / d}
$$

with $C_{m}, a>0$ independent of $d$. 
We see the following from $(28)-(34)$.

1. The smooth part for $f=1$ is just 1 . For general $f$, we will get a finite series of $M$ terms, which will have a corresponding effect on the smooth remainder $r_{d}$.

2. The boundary layers are exactly the 1-D ones for this special case, and are found along each of the four boundaries. For the case of general $f$, one obtains a sum of $M$ terms along each edge.

3. The functions $\tilde{Z}(\xi, \eta)$ contain the usual corner singularities $\tilde{r}^{1+m} \log \tilde{r}, m=0,1, \ldots$ where $\tilde{r}=\left(\xi^{2}+\eta^{2}\right)^{1 / 2}$. The corresponding singularities $Z(x, y)$ behave like $r^{1+m} \log r$, with $r=\left(\left(\frac{x}{d}\right)^{2}+\left(\frac{y}{d}\right)^{2}\right)^{1 / 2}$, and decay exponentially to 0 outside a square of side $O(d)$ at each corner. Hence, as $d \rightarrow 0$, the corner singularities become more and more "concentrated" at the corners, retaining essentially the same energy. (The behavior for plate and shell problems is different, see Section 5 below.)

For the approximation, we use the spaces $V_{N}=V\left(\Omega, \vec{p}_{N}, \mathcal{T}_{N}\right)$ where the polynomial degree $\vec{p}_{N}=p$ is chosen uniformly over elements in the mesh $\mathcal{T}_{N} \equiv \mathcal{T}_{p}$. We design $\mathcal{T}_{p}$ as follows. First, a layer of elements of width $k p d$ is used to approximate the term $u_{d, B L}$ given by (32). This is shown in Figure 16 for a quarter of the square (Scheme 1). Now to approximate $r^{\alpha}$ type singularities, one normally uses geometric mesh refinement as shown in Scheme 2. This suggests combining Scheme 1 and Scheme 2, to obtain Scheme 3. However, in our case, the singularities are "concentrated" in an $O(d)$ square, so that the geometric refinement must be carried out in this square. Hence, we arrive at Scheme 4. As our numerical experiments below indicate, Scheme 3 is not sufficient for proper convergence in the pointwise stresses near the point of singularity. Scheme 4 is the correct choice.

Let us define the mesh $\mathcal{T}_{N} \equiv \mathcal{T}_{p}$ for Scheme 4 more precisely. Let $I_{1}=(0, \kappa p d)$, $I_{2}=(\kappa p d, 1-\kappa p d), I_{3}=(1-\kappa p d, 1)$. Then first the tensor product mesh $\left\{\Omega_{i j}=I_{i} \times I_{j}\right\}$, $1 \leq i, j \leq 3$ is defined (Fig 17a). Next, each of the four corner squares is divided into two regions - e.g. $\Omega_{11}=R_{1} \cup R_{2}$, where $R_{1}=(0, \kappa d) \times(0, \kappa d)$ and $R_{2}=\Omega_{11} \backslash R_{1}$ (Fig 17b). A geometric mesh with ratio $\sigma, 0<\sigma<1$ and with $n=p$ layers, is constructed on $R_{1}$, following the guidelines of Babuska and Guo [3] (Fig 17c). The region $R_{2}$ is a transitional region connecting $R_{1}$ to the rest of the mesh on $\Omega$ (Fig $17 \mathrm{~d}$ ). Theorem 12 below, proved in [21], requires $R_{2}$ to contain at least $\nu(p)+1$ geometric layers, where $\nu(p)+1<\ln p /|\ln \sigma|$. However, in experiments, we found that a single transitional layer in this region was sufficient.

With the above definition of $\mathcal{T}_{p}$ (and hence of $V_{N}^{0}=V_{p}^{0}$ ) we obtain the following theorem.

Theorem 12 [21] Let $u_{d}$ be the solution of (15) - (16) with $f=1$ and $u_{d, p}$ be the corresponding finite element solution with $V_{p}^{0}$ as defined above. Then for any $s>0$ and any $\delta>0$, there exists a constant $C(s, \delta)$ independent of $d$ and $p$ such that

$$
\left\|u_{d}-u_{d, p}\right\|_{E, d, \Omega} \leq C(s, \delta) d^{1 / 2-\delta} p^{-s} .
$$




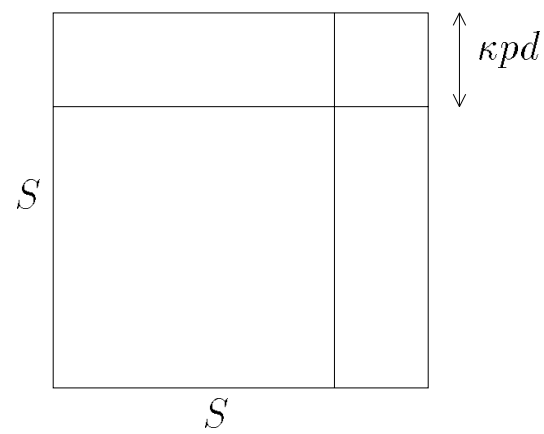

Scheme 1: BL-mesh

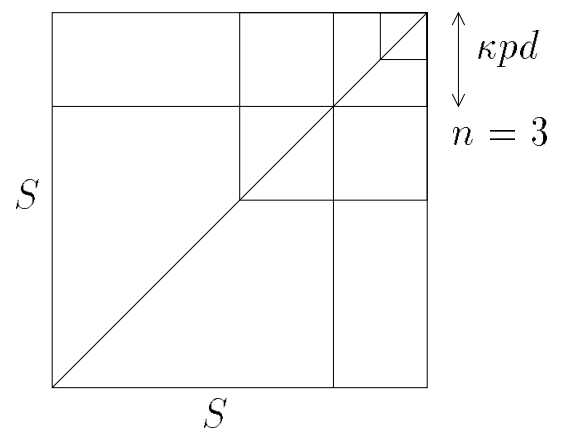

Scheme 3: BL-corner mesh

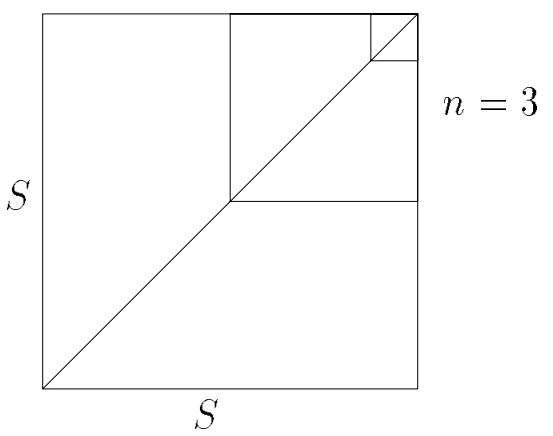

Scheme 2: Corner mesh

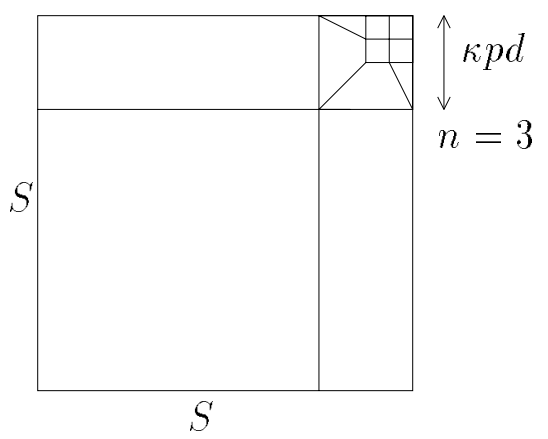

Scheme 4: BL-refined corner mesh

Figure 16: Meshes on a square. Scheme 1: Boundary layer mesh. Scheme D: Geometric refinement for $d=1$ with $n=3$ layers. Scheme 3: Union of Scheme 1 and Scheme 2. Scheme 4: Union of Scheme 1 and $d$-dependent geometric refinement with $n=3$ layers. 


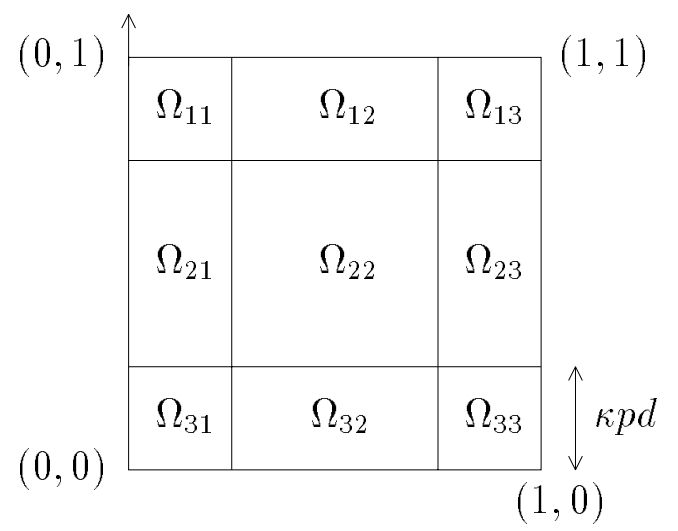

a)

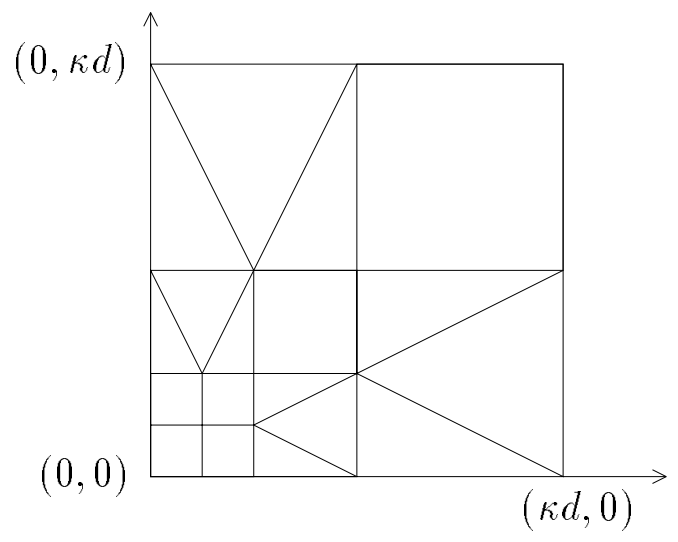

c)

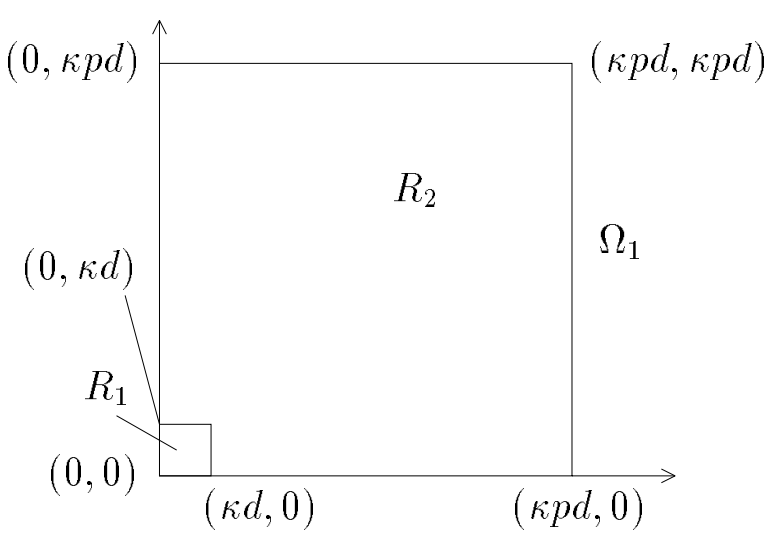

b)

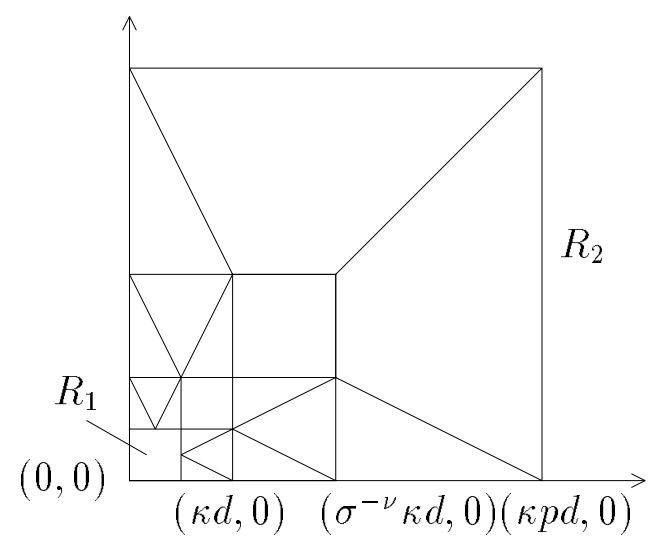

d)

Figure 17: Construction of the mesh on a square. a): Boundary layer mesh. b): The regions $R_{1}$ and $R_{2}$. c): Geometric refinement of $R_{1}$ with $\sigma=1 / 2$ and $n=3$ layers. d): Transitional region $R_{2}$ with $\sigma=1 / 2$ and $\nu(p)=2$. 
We now present some numerical results for our model problem (15) over the unit square with $f=1$. We consider the effect of using each of the four schemes described in Figure 16. (As in Section 3, we take the boundary layer mesh to be of thickness $p_{\max } d$ rather than $p d$ ). In Figure 18, we plot the relative error in the energy norm for $d=0.01$, 0.001. (Since the exact solution is not known, we use a finite element solution with several thousand degrees of freedom for comparison). The graphs indicate that Scheme 2, which does not have any boundary layer refinement, fails to give sufficient accuracy. All three schemes $(1,3,4)$ with boundary layer refinement exhibit an exponential decrease of the error, with Scheme 1 being the most efficient. This shows the necessity of boundary layer refinement.

Theorem 12 shows that as $d \rightarrow 0$, the energy norm error for the problem with $f=1$ decreases roughly as $O\left(d^{1 / 2}\right)$. This is observed for Scheme 4 from Figure 19.

Although Scheme 1 works best for the energy norm, the situation is different when pointwise derivatives of the solution are of interest. In Figure 20, we plot the relative error in the derivatives at the point $\left(x_{0}, y_{0}\right)=(1-d, 1-d)$ for $d=0.01,0.001$. Since this point is near the origin, an $O(d)$ geometric refinement is needed to resolve the $\left(\frac{r}{d}\right) \log \left(\frac{r}{d}\right)$ singularity here. It is observed that this is where the necessity of Scheme 4 comes in, which performs significantly better than any of the other schemes (Scheme 2 is not plotted at all, since it is even worse than Scheme 1). 

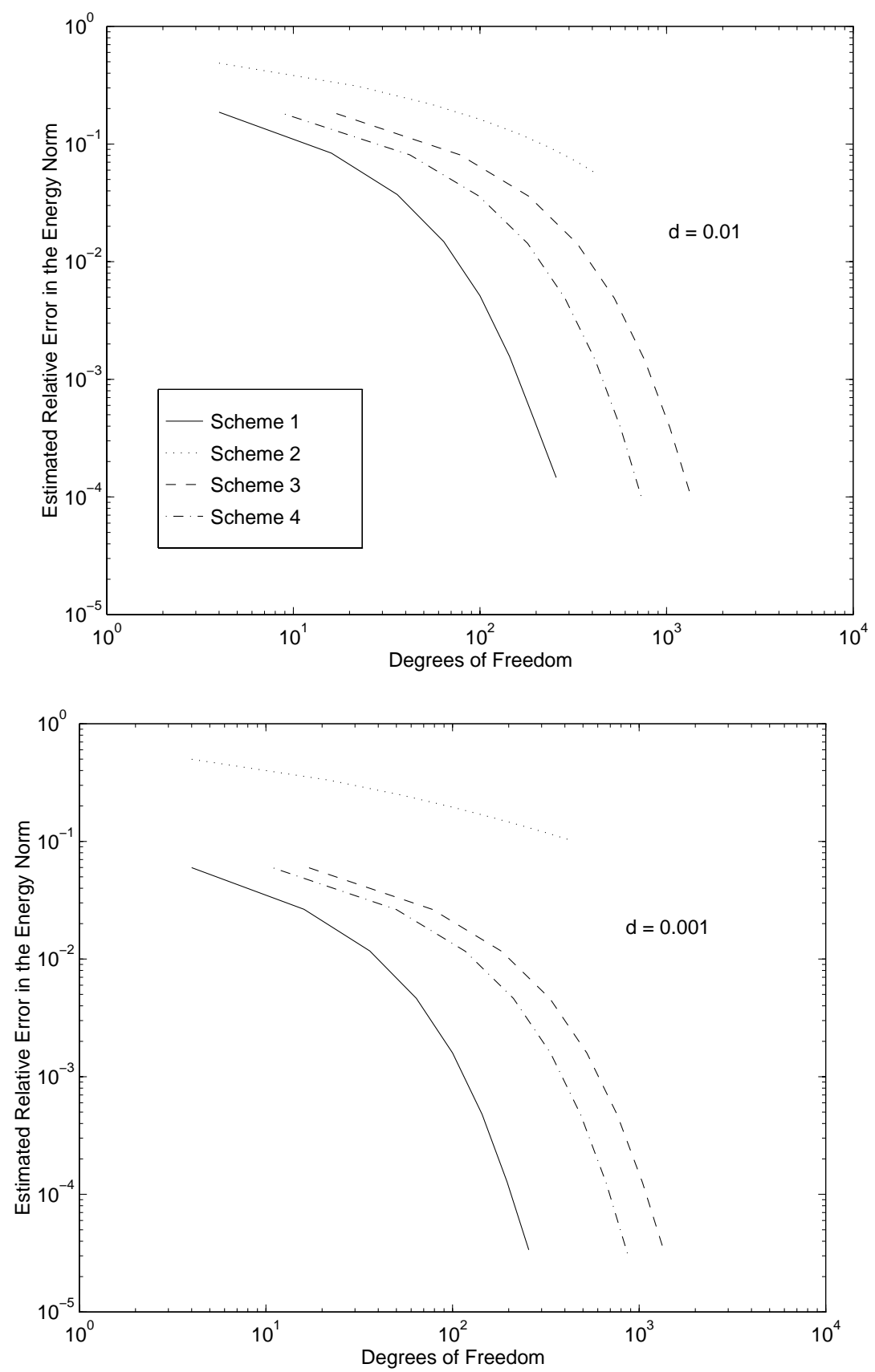

Figure 18: Energy norm convergence, $d=0.01, d=0.001$. 


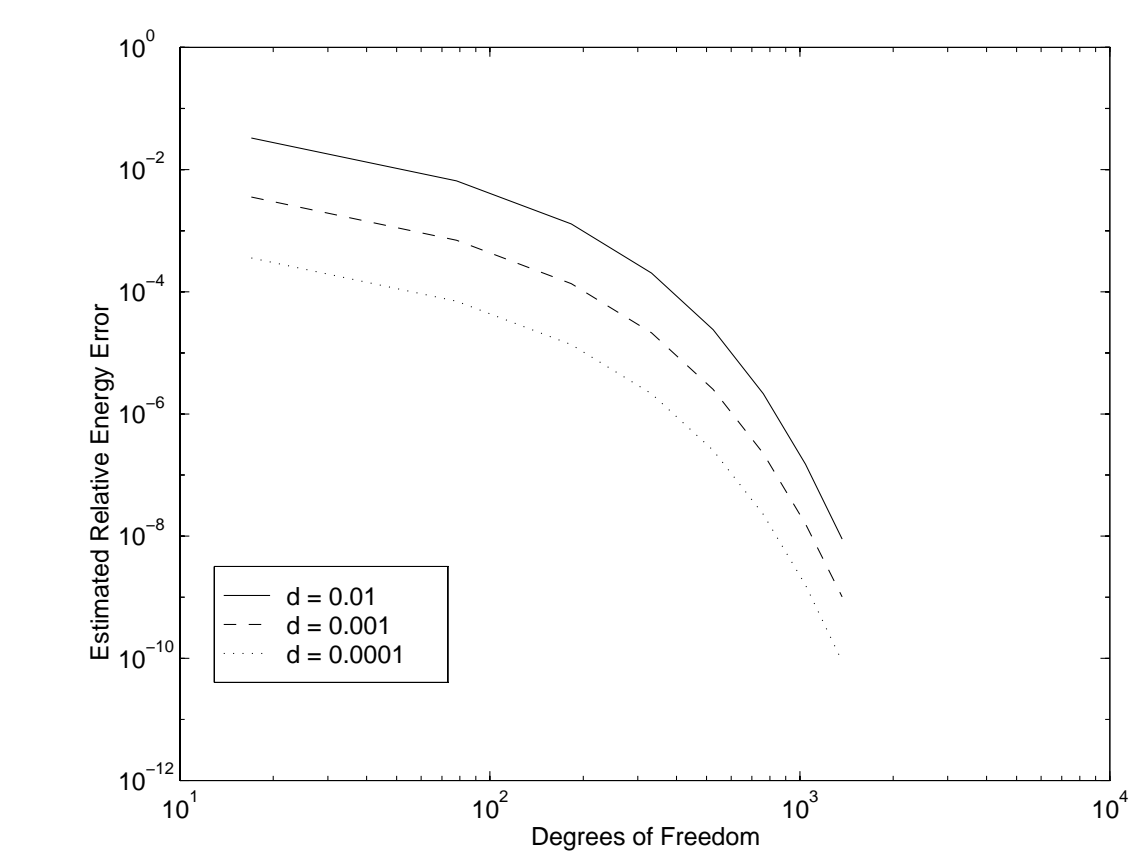

Figure 19: Energy norm convergence for Scheme 4, $d=0.01,0.001,0.0001$.

\subsection{Singularly perturbed problems over polygonal domains}

The guidelines of Section 4.1 can easily be extended to more general domains. For the heat transfer problem over a polygonal domain, it is easy to see what the mesh should look like near the boundary and in the vicinity of the vertices (Figure 21). This is based once again on a decomposition of the solution into various singular and boundary layer components, that may be found in [11].

Coming to the RM plate problem (24), (25) over a polygon, we notice an important difference from the heat transfer problem (15): For the limiting case $d=0$, the problem now reduces to the biharmonic, which will have its own $r^{\alpha}$ type singularities ( $\alpha$ possibly complex), in an $O(1)$ region at each vertex. Both of these corner singularities can be approximated at a robust exponential rate, provided the geometric mesh refinement is now initiated in an $O(1)$ rather than $O(d)$ neighborhood of the corner. In addition, boundary layers would also have to be resolved. For instance, in the case of a square, the recommendation would now be to superimpose Scheme 2 on Scheme 4. Once again, locking effects also have to be considered - however, as shown in [15], [4], the use of high $p$, as recommended here, is an effective way of controlling this phenomenon. 

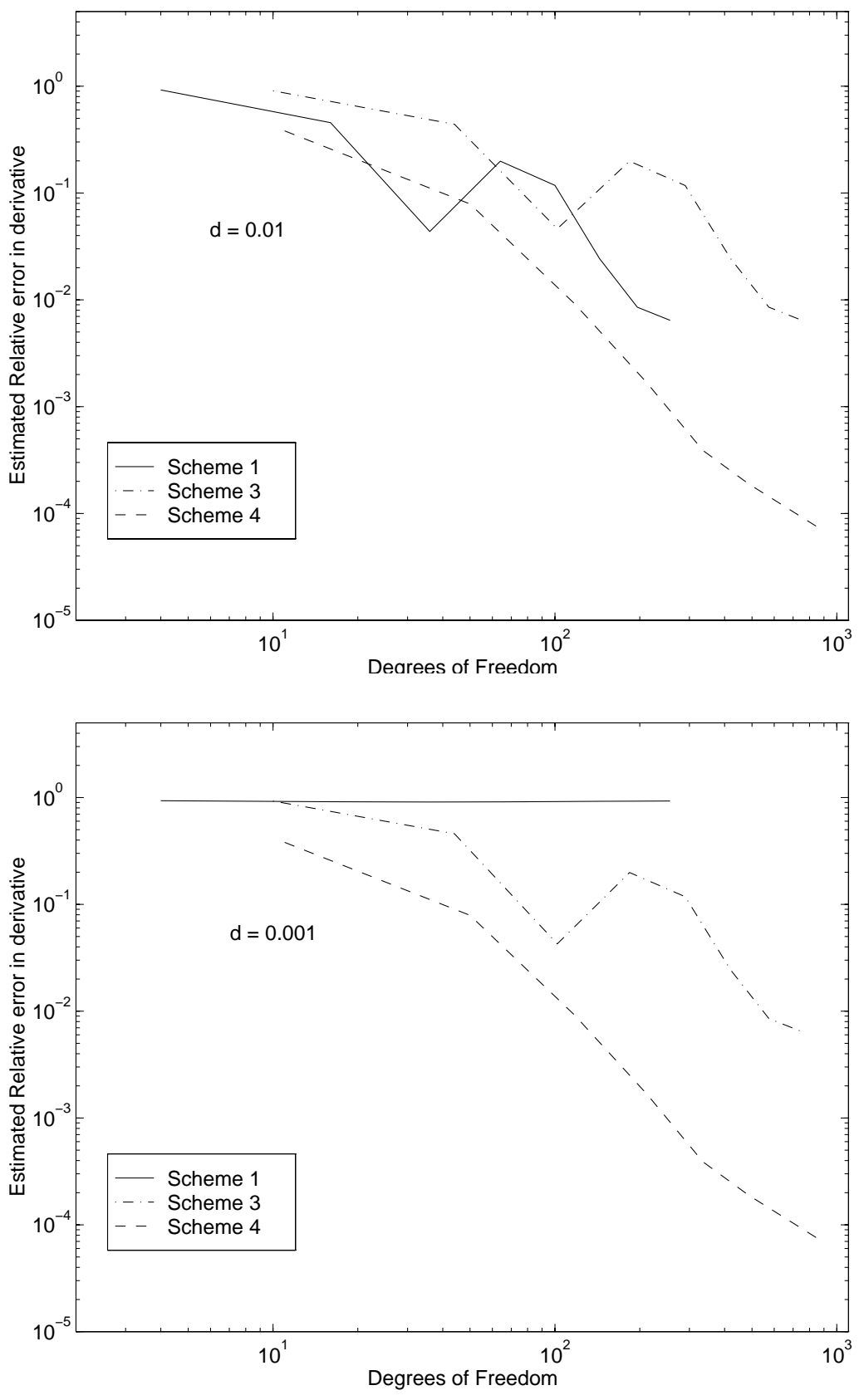

Figure 20: Pointwise derivative convergence, $d=0.01, d=0.001$. 


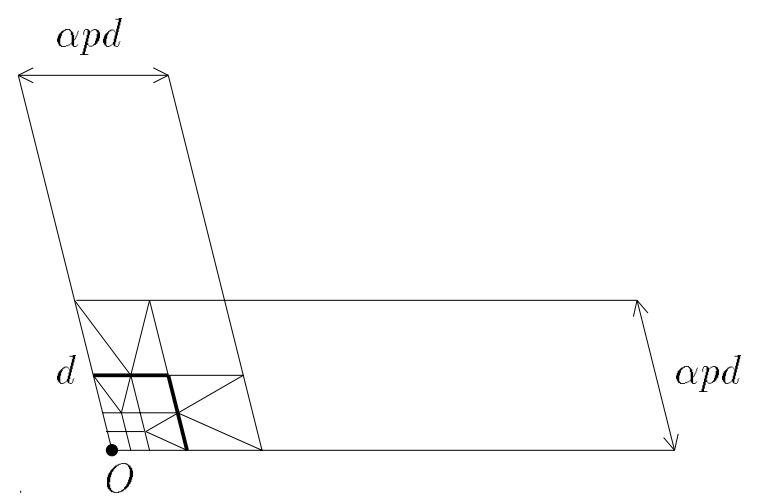

Figure 21: $h p$-mesh in the vicinity of a vertex of a general polygon diffusion problem

\section{$5 \quad$ Mesh design for $h p$ FE approximation of shells}

Shells are thin, three-dimensional solids with small thickness $t$ and midsurface $S \subset \mathbb{R}^{3}$. We assume that $S$ is parametrized by a chart $\varphi$, i.e.

$$
S=\varphi(\Omega), \quad \Omega \subset \mathbb{R}^{2} \text { polygon }
$$

The deformation of $S$ is normally described by a shell model. Two broad classes of shell models are those of Koiter type and those admitting shear deformation, to which we refer as Naghdi models. The analysis of the structure of the shell boundary layers and corner singularities is to some extent open; however, the length scales at which boundary layers may occur have been investigated for some time (see the book of Rutten [23] and the references there).

We present the main results about shell boundary layers:

1. The shell models of Koiter type (i.e. involving 4th-order derivations of the normal displacement) exhibit in general several boundary layers of the form (1). One always has a layer with $d=\sqrt{t}$ and $\alpha=1+i$ due to the bending-membrane coupling (also referred to as "simple edge effect"). In addition, depending on the geometry of the midsurface $S$, further layers may arise (see Figure 22)

(a) S is elliptic.

In the K shell model, only the $\sqrt{t}$ layer appears.

(b) S is parabolic.

Depending on the alignment of $\partial \Omega$ with the principal curvature lines, an additional layer of length scale $d=\sqrt[4]{t}$ can appear (e.g. in Figure 23, along the axes of the cylinder).

(c) S is hyperbolic.

There are now two families of characteristic lines, along which the curvatures are constant. In the limit $t \rightarrow 0$, the system describing the membrane deformation is hyperbolic ([13]) and point loads and corner singularities propagate 
along these characteristics (see Figure 24). At small, positive thickness, there will be layers of scale $d=\sqrt{t}$ as above at $\partial \Omega$, and additionally layers of scale $d=O(\sqrt[3]{t})$ normal to characteristics passing through points where the bendings and/or the boundary $\partial \Omega$ is not smooth.

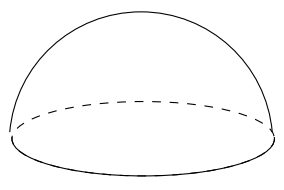

a) elliptic

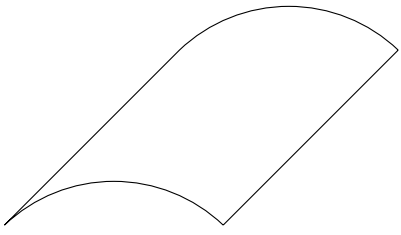

b) parabolic

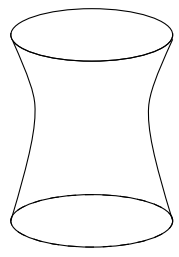

c) hyperbolic

Figure 22: Midsurfaces $S$ of shells

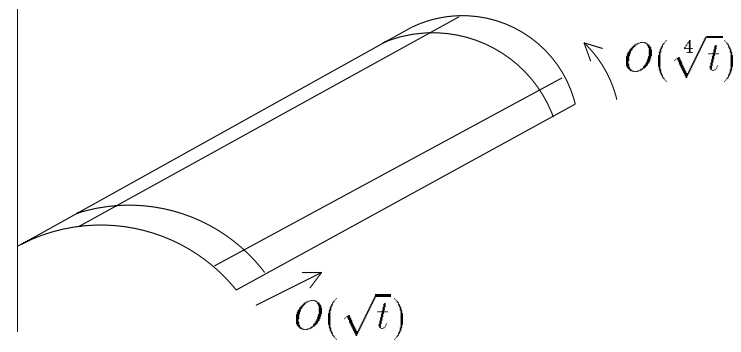

Figure 23: Boundary layers in parabolic shells

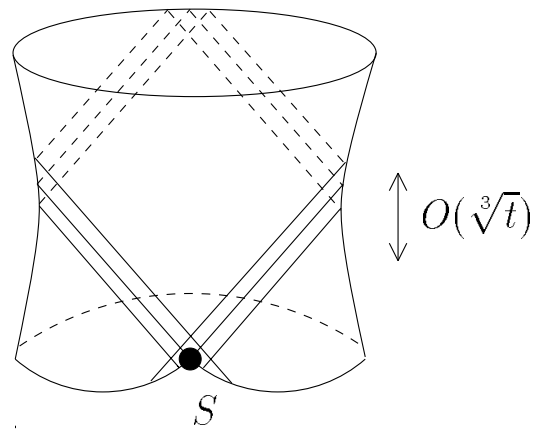

Figure 24: Interior layers coupled with singularity in hyperbolic shells generated by reentrant corner $S$

2. In models allowing for shear deformation (such as the Naghdi type models), the above layers will appear and, regardless of the geometry of $S$, there will be an additional layer of length scale $d=O(t)$, as in the Reissner-Mindlin plate. 
As we observed already for heat conduction and plate bending problems, failure to resolve the $O(t)$ layer in the Naghdi shell models may not be apparent in the energy norm, but will show up when pointwise stresses and moments are of interest. In contrast to that, the layers of the longer scales $d=\sqrt{t}, \sqrt[3]{t}, \sqrt[4]{t}$ often carry a substantial percentage of the total deformation energy and must be resolved in order to get meaningful approximations. Therefore we obtain, based on our available results in the 1 -D and 2-D setting, the following recommendations for mesh design of shells:

1. For the $K$-models one should use one boundary layer element of thickness $\kappa p d$ with $d=\sqrt{t}$. In addition, if the shells are very thin, $\left(t / \operatorname{diam}(S)<10^{-3}\right)$, further boundary layer elements of width $\kappa p d$ with $d=\sqrt[4]{t}$ should be added if $S$ is not elliptic. For very thin shells of hyperbolic type, there will be internal layers with $d=O(\sqrt[3]{t})$ along characteristic lines through corners or points of nonsmoothness of the data.

2. In models of Naghdi type (i.e. admitting shear deformation), one further layer of width $k p d$ with $d=t$ should be added in addition to the $O(\sqrt{t}), O(\sqrt[3]{t}), O(\sqrt[4]{t})$ layers mentioned in 1.

We remark finally that in the presence of corners (i.e. $\partial \Omega$ nonsmooth), proper geometric mesh refinement towards corners has to be done. This has to be anisotropic as well, if the layers meeting at a corner have different length scales (see Figure 25). Here the corner singularity also "propagates" along lines of constant curvature (shown dashed in Figures 24 and 25). Questions of how best to design the mesh to resolve the boundary layers in the presence of such singularities, and in the presence of locking have only been partially answered (see e.g. [26]).

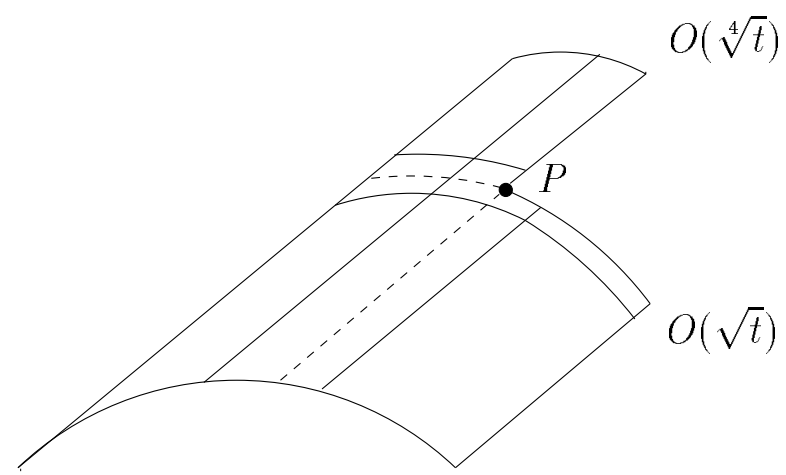

Figure 25: Parabolic shell with reentrant corner $P$ 


\section{References}

[1] D. N. Arnold and R. S. Falk, Asymptotic analysis of the boundary layer for the Reissner-Mindlin plate model, SIAM J. Math. Anal., Vol. 27, No. 2, pp. 486-514, 1996.

[2] D. N. Arnold and R. S. Falk, Edge effects in the Reissner-Mindlin plate model, Analytic and Computational Models of Shells, A.S.M.E., New York, 1989.

[3] I. Babuska and B. Guo, The h-p version of the finite element method. Part I : The basic approximation results, Computational Mechanics (1986) 1, 21-41.

[4] I. Babuska and Li, Hierarchic Modelling of Plates, Computers and Structures 40 : 419-430, 1991

[5] I. A. Blatov and V. V. Strygin, On estimates best possible in order in the Galerkin finite element method for singularly perturbed boundary value problems, Russian Acad. Sci. Dokl. Math. 47 (1993), 93-96.

[6] V.F. Butuzov, The asymptotic properties of the equation $\mu^{2} \Delta u-\kappa^{2}(x, y) u=f(x, y)$ in a rectangle, Differentsial'nye Uravneniya, 99:1274-1279, 1973.

[7] W. Eckhaus, Boundary layers in linear elliptic singular perturbation problems, SIAM Review, 14:225-271,1972.

[8] C. Schwab, Mesh design for structural plates, ESRD Technical Brief, January 1995.

[9] E. C. Gartland, Uniform high-order difference schemes for a singularly perturbed two-point boundary value problem, Math. Comp., 48:551-564, 1987.

[10] H. Han and R. B. Kellogg, Differentiability properties of solutions of the equation $-\varepsilon^{2} \Delta u+r u=f(x, y)$ in a square, SIAM J. Math Anal., Vol 21, No. 2, pp. 394-408, March 1990 .

[11] R. B. Kellogg, Boundary layers and corner singularities for a self adjoint problem, in Boundary Value Problems and Integral Equations in Nonsmooth Domains, M.Costabel, M.Dauge and S.Nicaise (Eds.), Lecture Notes in Pure and Applied Mathematics vol. 167, Marcel Dekker, New York 1995, 121-149.

[12] J. M. Melenk, A note on robust exponential convergence of FEM for problems with boundary layers, Report 96-06, SAM, ETH Zürich, May 1996.

[13] J. Piila, Characterization of the membrane Theory of a hyperbolic shell, Math. Mod. Meth. Appl. Sci. (1996).

[14] J. Pitkäranta, Y. Leino, O. Ovaskainen and J. Piila, Shell deformation states and the finite element method: A benchmark study of cylindrical shells, Research Report A333, Institute of Math, Helsinki University of Technology, May 1994. 
[15] M. Suri, I. Babuska and C. Schwab, Looking effects in the finite element approximation of plate models, Math. Comp. 64 (1995), pp. 461-482.

[16] Shishkin, G. I., Grid approximation of singularly perturbed boundary value problems with a regular boundary layer, Soviet J. Numer. Anal. Math. Modelling, 4 (1989), pp. $397-417$

[17] M. Suri, Analytical and computational assessment of locking in the hp finite element method, Comp. Mech. Engg. 133 (1996), pp. 347-371.

[18] C. Schwab and M. Suri, The $p$ and hp versions of the finite element method for problems with boundary layers, Math. Comp., to appear, 1996.

[19] C. Schwab and M. Suri, Locking and boundary layer effects in the finite element approximation of the Reissner-Mindlin plate model, Proc. Symp. Appl. Math.,48:367$371,1994$.

[20] R. Vulanovic, D. Herceg and N. Petrovic, On the extrapolation for a singularly perturbed boundary value problem, Computing, 36:69-79, 1986.

[21] C. A. Xenophontos, The hp version of the finite element method for singularly perturbed problems, Ph.D. Dissertation, UMBC, 1996.

[22] H.G. Roos, M. Stynes and L. Tobiska, Numerical Methods for Singularly Perturbed Differential Equations, Springer Verlag (1996).

[23] H.S. Rutten, Theory and Design of Shells on the Basis of Asymptotic Analysis, Rutten and Kruisman, Consulting Engineers, Holland 1973.

[24] I. Babuska \& C. Schwab, A-posteriori error estimation for hierarchic models of elliptic problems on thin domains, SIAM J. Numer. Anal. 33 (1996), pp. 221-246.

[25] C. Schwab: Hierarchic Modelling in Mechanics by the hp-FEM, Lecture Notes for the VIIth EPSRC Summer School on Numerical Analysis, Leicester UK, Aug. 1996 (to appear with Oxford Univ. Press 1997).

[26] H. Hakula, Y. Leino and J. Pitkäranta: Scale resolution, locking and high-order numerical modelling of shells, Comp. Meth. Appl. Mech. Engg. 133 (1996) 157-182. 